\title{
Precise Infrared Radial Velocities From Keck/ nirspec and the Search for Young Planets
}

\section{Citation}

Bailey, John I., Russel J. White, Cullen H. Blake, Dave Charbonneau, Travis S. Barman, Angelle M. Tanner, and Guillermo Torres. 2012. "PRECISE INFRARED RADIAL VELOCITIES FROM KECK/ NIRSPEC AND THE SEARCH FOR YOUNG PLANETS." The Astrophysical Journal 749 (1): 16. https://doi.org/10.1088/0004-637x/749/1/16.

\section{Permanent link}

http://nrs.harvard.edu/urn-3:HUL.InstRepos:41397388

\section{Terms of Use}

This article was downloaded from Harvard University's DASH repository, and is made available under the terms and conditions applicable to Other Posted Material, as set forth at http:// nrs.harvard.edu/urn-3:HUL.InstRepos:dash.current.terms-of-use\#LAA

\section{Share Your Story}

The Harvard community has made this article openly available. Please share how this access benefits you. Submit a story. 


\title{
PRECISE INFRARED RADIAL VELOCITIES FROM KECK/NIRSPEC AND THE SEARCH FOR YOUNG PLANETS
}

\author{
John I. Bailey III ${ }^{1,6}$, Russel J. White ${ }^{2}$, Cullen H. Blake ${ }^{3}$, Dave Charbonneau ${ }^{4}$, \\ Travis S. Barman ${ }^{5}$, Angelle M. TANner ${ }^{2,7}$, ANd Guillermo Torres ${ }^{4}$ \\ ${ }^{1}$ Physics Department, University of Alabama Huntsville, 301 Sparkman Drive, 201B Optics Building, Huntsville, AL 35899, USA; baileyji@ umich.edu \\ ${ }^{2}$ Department of Physics and Astronomy, Georgia State University, 29 Peachtree Center Avenue, Science Annex, \\ Suite 400, Atlanta, GA 30303, USA; white@ chara.gsu.edu \\ ${ }^{3}$ Department of Astrophysical Sciences, Princeton University, Peyton Hall, Ivy Lane, Princeton, NJ 08544, USA \\ ${ }^{4}$ Harvard-Smithsonian Center for Astrophysics, 60 Garden Street, Cambridge, MA 02138, USA \\ ${ }^{5}$ Lowell Observatory, 1400 West Mars Hill Road, Flagstaff, AZ 86001, USA \\ Received 2011 June 17; accepted 2012 January 26; published 2012 March 16
}

\begin{abstract}
We present a high-precision infrared radial velocity (RV) study of late-type stars using spectra obtained with NIRSPEC at the W. M. Keck Observatory. RV precisions of $50 \mathrm{~m} \mathrm{~s}^{-1}$ are achieved for old field mid-M dwarfs using telluric features for wavelength calibration. Using this technique, 20 young stars in the $\beta$ Pic (age $\sim 12 \mathrm{Myr}$ ) and TW Hya (age $\sim 8 \mathrm{Myr}$ ) Associations were monitored over several years to search for low-mass companions; we also included the chromospherically active field star GJ 873 (EV Lac) in this survey. Based on comparisons with previous optical observations of these young active stars, RV measurements at infrared wavelengths mitigate the RV noise caused by star spots by a factor of $\sim 3$. Nevertheless, star spot noise is still the dominant source of measurement error for young stars at $2.3 \mu \mathrm{m}$, and limits the precision to $\sim 77 \mathrm{~m} \mathrm{~s}^{-1}$ for the slowest rotating stars $\left(v \sin i<6 \mathrm{~km} \mathrm{~s}^{-1}\right)$, increasing to $\sim 168 \mathrm{~m} \mathrm{~s}^{-1}$ for rapidly rotating stars $\left(v \sin i>12 \mathrm{~km} \mathrm{~s}^{-1}\right)$. The observations reveal both GJ 3305 and TWA 23 to be single-lined spectroscopic binaries; in the case of GJ 3305, the motion is likely caused by its 0 '. 09 companion, identified after this survey began. The large amplitude, short-timescale variations of TWA 13A are indicative of a hot Jupiter-like companion, but the available data are insufficient to confirm this. We label it as a candidate RV variable. For the remainder of the sample, these observations exclude the presence of any "hot" $(P<3$ days $)$ companions more massive than $8 M_{\text {Jup }}$ and any "warm" $(P<30$ days $)$ companions more massive than $17 M_{\text {Jup }}$, on average. Assuming an edge-on orbit for the edge-on disk system AU Mic, these observations exclude the presence of any hot Jupiters more massive than $1.8 M_{\text {Jup }}$ or warm Jupiters more massive than $3.9 M_{\text {Jup }}$.
\end{abstract}

Key words: methods: data analysis - planets and satellites: formation - stars: pre-main sequence - starspots techniques: radial velocities - techniques: spectroscopic

\section{INTRODUCTION}

The remarkable discovery of extrasolar planets orbiting sun-like stars in 1995 was accomplished by utilizing novel techniques that improved the radial velocity $(\mathrm{RV})$ precision by two orders of magnitude (from $\sim 1 \mathrm{~km} \mathrm{~s}^{-1}$ to $10 \mathrm{~m} \mathrm{~s}^{-1}$ ), which was at last sufficient to search for the slight gravitational wobble induced by an orbiting gas giant planet upon its host star (Walker et al. 1995; Mayor \& Queloz 1995; Marcy \& Butler 1996; Cochran et al. 1997; but see also Latham et al. 1989). Since then an extensive international effort has begun to determine the ubiquity and basic properties of these initially elusive objects. Based on a modest extrapolation of current findings, we now know that roughly $12 \%$ of Sun-like stars (F,G, and K spectral type) harbor gas giant planets within $30 \mathrm{AU}$ (e.g., Butler et al. 2006; Udry et al. 2007; Cumming et al. 2008; Wright et al. 2010; Howard et al. 2010). While the masses of these planets are similar to the gas giants in our solar system (i.e., Jupiter and Saturn), their orbital properties are in many ways radically different. Some orbit their host star in just a few days time (the "hot Jupiters"), while others have highly eccentric orbits. Planetlike companions have even been directly imaged at separations of more than $100 \mathrm{AU}$ from their host star (Kalas et al. 2008).

\footnotetext{
6 Current address: Department of Astronomy, University of Michigan, 830 Dennison Building, 500 Church Street, Ann Arbor, MI 48109, USA.

7 Current address: Department of Physics and Astronomy, Mississippi State University, 355 Lee Boulevard, Hilbun Hall, Starkville, MS 39762.
}

The dramatically different properties of extrasolar planets compared to those in our solar system forced theorists to reconsider the standard paradigm for gas giant planet formation. Now, planet formation scenarios generally fall into two categories: core accretion (Mizuno et al. 1980) and disk instabilities (Boss 1997). Core accretion is the favored mechanism for producing giant planets with large core masses (e.g., Sato et al. 2005) and can naturally explain the enhanced frequency of planets around metal-rich stars (e.g., Robinson et al. 2006). However, at wide separations ( $\gtrsim 10 \mathrm{AU})$ the timescale for planet formation via core accretion (Pollack et al. 1996; Alibert et al. 2005) is longer than the typical disk dissipation timescale $(\lesssim 10 \mathrm{Myr}$; Haisch 2001; Briceño et al. 2001; Carpenter et al. 2006; Pascucci et al. 2006); it is thus an unlikely formation mechanism for the planets in very wide orbits about Fomalhaut and HR 8799 (Marois et al. 2008; Kalas et al. 2008). Disk instabilities, on the other hand, can form planets much more quickly $(\lesssim 1 \mathrm{Myr})$, well before circumstellar disks are dispersed (Boss 1997, 2004; Mayer et al. 2002; Meru \& Bate 2010; but see also Stamatellos \& Whitworth 2008; Meru \& Bate 2011). This mechanism also works nearly as efficiently around low-mass stars, with sufficiently massive disks, and seems a more plausible scenario for forming the known massive planets orbiting $\mathrm{M}$ dwarfs (Boss 2006). Of course, it is possible that both scenarios operate or dominate within different regimes of stellar and/or disk mass.

In addition to the uncertainties surrounding planet formation, it is also unclear how the orbits of newly formed planets dynamically evolve. Since giant planets are believed to form 
beyond the snow line (e.g., Kennedy \& Kenyon 2008), inward orbital migration must occur in many systems to explain the wealth of short-period planets; roughly $10 \%$ of gas giant planets have separations less than 0.1 AU (Butler et al. 2006). Interactions with disk material are widely believed to be the most efficient mechanism for inward migration (e.g., Goldreich \& Tremaine 1980; Lin et al. 1996). If most gas giants migrate via this process, then the hot Jupiters must become "hot" within $\sim 10$ Myr after formation, prior to disk dissipation. However, planet-disk interactions typically circularize orbits and maintain spin-orbit alignment with the star, so other processes such as dynamical scattering with other planets (Adams \& Laughlin 2003; Raymond et al. 2011) or a nearby star (Malmberg et al. 2007; Malmberg \& Davies 2009) may be needed to produce the broad extrasolar planet eccentricity distribution and the spin—orbit misalignments of many transiting planets (e.g., Pont et al. 2009). These dynamical interactions could take many hundreds of millions of years (e.g., see review by Lubow \& Ida 2010).

One direct way to begin constraining the mechanisms and timescales of planet formation and migration is to search for planets around stars with ages of $\lesssim 10$ Myr, or essentially immediately after planets could have formed. Direct imaging searches for planets have been more successful at this recently than RV searches. Several massive Jupiter-like companions have been directly imaged around stars with these ages, though at somewhat wide separations (10 to many 100 s of AU; e.g., Neuhäuser et al. 2005; Lagrange et al. 2010; Ireland et al. 2011); these important discoveries are helping to motivate future highcontrast imaging facilities and the science achievable with them (e.g., Macintosh et al. 2008; Kataria \& Simon 2010). In many cases, however, planetary status relies upon comparisons with poorly tested evolutionary models, and the wide separations offer little constraint on the migration mechanism responsible for forming hot Jupiter-like planets.

Planets identified via RV variations, on the other hand, yield immediate-mass lower limits $(m \sin i)$ and preferentially find massive short-period planets which most likely have migrated. However, two universal properties of young stars inhibit measuring their RV precisely at optical wavelengths, and consequently they are often excluded in high-precision RV surveys for planets. The first of these is that young Sun-like stars are much cooler while young and thus fainter at optical wavelengths. A 1 solar mass star, for example, has a spectral type of $\sim \mathrm{K} 5$ at 10 Myr (Baraffe et al. 1998; Siess et al. 2000). This, coupled with the fact that all star-forming regions are further away than $100 \mathrm{pc}$, means that most young stars are simply too faint for high-precision RV work at optical wavelengths. The second of these is that young stars are often modestly to rapidly rotating. This rotation broadens stellar absorption features and, even more perniciously, generates chromospheric activity and a spotted stellar surface. Several pioneering RV studies of young spotted stars have shown that their presence can induce low amplitude, periodic RV shifts, and thus mimic the effect of one or more orbiting planets (Queloz et al. 2001; Paulson \& Yelda 2006; Huerta et al. 2008; Prato et al. 2008; Reiners et al. 2010; Crockett et al. 2011; Mahmud et al. 2011); amplitudes of 200-700 $\mathrm{m} \mathrm{s}^{-1}$ have been observed. In a couple cases, Jupiterlike companions have been reported orbiting young stars based on low-amplitude $\left(<1 \mathrm{~km} \mathrm{~s}^{-1}\right) \mathrm{RV}$ variations (Setiawan et al. 2008; Hernán-Obispo et al. 2010), but subsequent observations indicate that these too were misidentifications caused by star spots (Huélamo et al. 2008; Figueira et al. 2010).
One promising way to overcome these observational challenges is to observe at infrared wavelengths. As has been demonstrated, modern infrared spectrographs utilizing molecular gas cells are able to obtain RV precisions comparable to very stable optical facilities (e.g., Bean et al. 2010). This wavelength range is advantageous for young stars on cool convective tracks since it is much closer to the peak of their energy distribution, thereby improving observing efficiency. More significantly, at these wavelengths the contrast between the photosphere and cool star spots is reduced; this is confirmed by the decline in the photometric variability of spotted stars with wavelength (e.g., Herbst et al. 1994). Since the RV "noise" induced by star spots is directly proportional to the amplitude of a star's photometric variability (Saar \& Donahue 1997), the diminished variability at longer wavelength observations should help mitigate the noise of star spots. Observations of young spotted stars at both optical and infrared wavelengths appear to confirm this prediction (Prato et al. 2008; Huélamo et al. 2008; Mahmud et al. 2011).

Motivated by the prospects for obtaining high-precision RV measurements of young stars at infrared wavelengths, in late 2004 we began an ambitious observational program at the W. M. Keck Observatory (PI: R. White) to search for young planets. Here we present initial results. In Section 2, we describe the sample selection and in Section 3 we summarize the acquisition of infrared spectra. In Sections 4 and 5, we describe our method for optimally extracting the spectra and for modeling the spectra to determine precise RVs. The results of this survey, which include the discovery of two spectroscopic binaries and one candidate hot Jupiter, are highlighted in Section 6. In Section 7, we determine detection limits for the ensemble sample via Monte Carlo simulations and quantify the advantage infrared RVs measurements have over optical measurements for young stars. Our overall findings are summarized in Section 8.

\section{THE OBSERVATIONAL SAMPLE: YOUNG STARS AND COMPARISON FIELD STARS}

Although our entire young star survey includes stars from both star-forming regions and nearby loose associations, in this first paper we focus only on young stars in the $\beta$ Pic and TW Hydrae Associations, which have ages of $\sim 12 \mathrm{Myr}$ and $\sim 8 \mathrm{Myr}$, respectively (Torres et al. 2008). The high-quality spectra of these nearby, systematically brighter stars were our initial focus as we developed techniques for precise RV measurements. The selection criteria for targets within these associations were that the stars have no known companions within 2 ". 0 , be somewhat slowly rotating ( $v \sin i \lesssim 15 \mathrm{~km} \mathrm{~s}^{-1}$, if known), show no signs of accretion (e.g., TW Hya itself was excluded), and have an $\mathrm{M}$ spectral type. The spectral-type criterion was adopted to ensure that stars have strong ${ }^{12} \mathrm{CO} \mathrm{R}$-branch lines in their spectra, from which RVs are measured. Stars were selected from the membership lists assembled in Zuckerman \& Song (2004); nine stars from the $\beta$ Pic Association and nine stars from the TW Hydrae Association met these criteria. Wide companions to two of these stars have spectral types just slightly earlier than M, AG Tri A (K6) and TWA 9A (K5), and were therefore also included since their acquisition overhead would be minimal. Spectroscopic observations of a few of these stars (GJ 871.1 B, TWA 12 , TWA 23 ) revealed that they were more rapidly rotating than our initial selection criterion. We nevertheless continued to observe these stars in order to investigate empirically how the precision degrades with projected rotational velocity increasing.

After our observational program began, one of our targets, GJ3305, was discovered to be a 0.'093 binary (Kasper et al. 
Table 1

Observed Sample

\begin{tabular}{|c|c|c|c|c|c|c|c|c|}
\hline $\begin{array}{l}\text { Star } \\
\text { Name }\end{array}$ & $\mathrm{SpT}$ & Reference & $\begin{array}{c}K \\
(\mathrm{mag})\end{array}$ & $\begin{array}{l}\text { Mass } \\
\left(M_{\odot}\right)\end{array}$ & $\begin{array}{l}\text { Period } \\
\text { (days) }\end{array}$ & Reference & $\begin{array}{c}v \sin i \\
\left(\mathrm{~km} \mathrm{~s}^{-1}\right)\end{array}$ & Reference \\
\hline \multicolumn{9}{|c|}{ Single field stars } \\
\hline GJ 628 & M3.5 & H94 & 5.07 & 0.30 & $\ldots$ & $\ldots$ & $<1.1$ & D98 \\
\hline GJ 725A & M3 & H94 & 4.43 & 0.37 & $\ldots$ & $\ldots$ & $<2.8$ & D98 \\
\hline GJ 725A & M3.5 & H94 & 5.00 & 0.30 & $\ldots$ & $\cdots$ & $<2.8$ & D98 \\
\hline \multicolumn{9}{|c|}{ Chromospherically active field stars } \\
\hline GJ 873 & M3.5 & H94 & 5.30 & 0.30 & 4.38 & M95 & $\ldots$ & $\ldots$ \\
\hline \multicolumn{9}{|c|}{$\beta$ Pic stars } \\
\hline AU Mic & M0 & H96 & 4.53 & 0.73 & 4.87 & M95 & 8.5 & S07 \\
\hline AG Tri A & K6 & S03 & 7.08 & 0.94 & 13.7 & N07 & $\ldots$ & $\ldots$ \\
\hline AG Tri B & M0 & S03 & 7.92 & 0.73 & $\ldots$ & $\ldots$ & $\ldots$ & $\ldots$ \\
\hline GJ 182 & M0 & H96 & 6.26 & 0.73 & 4.56 & M95 & $\ldots$ & $\ldots$ \\
\hline GJ 3305 & M0.5 & H96 & 6.41 & 0.67 & 6.10 & F06 & 5.3 & S07 \\
\hline GJ 799 A & M4.5 & H96 & 5.69 & 0.16 & $\ldots$ & $\ldots$ & 10.6 & S07 \\
\hline GJ 799 B & M4 & H96 & 5.69 & 0.22 & $\ldots$ & $\ldots$ & 17.0 & S07 \\
\hline GJ $871.1 \mathrm{~A}$ & M4 & S02 & 6.93 & 0.22 & 2.355 & M10 & 14.0 & S07 \\
\hline GJ $871.1 \mathrm{~B}$ & M4.5 & S02 & 7.79 & 0.16 & $\ldots$ & $\ldots$ & 24.3 & S07 \\
\hline HIP 12545 & M0 & S03 & 7.07 & 0.73 & 1.25 & M10 & 9.3 & S07 \\
\hline \multicolumn{9}{|c|}{ TW Hya stars } \\
\hline TWA 7 & M1 & W99 & 6.90 & 0.60 & 5.05 & $\mathrm{LC} 05$ & $<5$ & S07 \\
\hline TWA 8A & M2 & W99 & 7.43 & 0.51 & 4.65 & $\mathrm{LC} 05$ & $<5$ & S07 \\
\hline TWA 8B & M5 & W99 & 9.01 & 0.12 & 0.78 & $\mathrm{LC} 05$ & 11.2 & S07 \\
\hline TWA 9A & K5 & W99 & 7.85 & 1.01 & 5.10 & LC05 & 11.3 & S07 \\
\hline TWA 9B & M1 & W99 & 9.15 & 0.60 & 3.98 & $\mathrm{LC} 05$ & 8.4 & S07 \\
\hline TWA 11B & M2.5 & W99 & 8.35 & 0.44 & $\ldots$ & $\ldots$ & 12.1 & S07 \\
\hline TWA 12 & M2 & S99 & 8.05 & 0.51 & 3.28 & LC05 & 16.2 & S07 \\
\hline TWA $13 \mathrm{~A}$ & M1 & S99 & 7.49 & 0.60 & 5.56 & LC05 & 10.5 & S07 \\
\hline TWA 13B & M2 & S99 & 7.46 & 0.51 & 5.35 & LC05 & 14.8 & S07 \\
\hline TWA 23 & M1 & S03 & 7.75 & 0.60 & $\ldots$ & $\ldots$ & $\ldots$ & $\ldots$ \\
\hline
\end{tabular}

References-D98: Delfosse et al. (1998); F96: Feigelson et al. (2006); H94: Henry et al. (1994); H96: Hawley et al. (1996); LC05: Lawson \& Crause (2005); M95: Mathioudakis et al. (1995); N07: Norton et al. (2007); S02: Song et al. (2002); S03: Song et al. (2003); S07: Sholtz et al. (2007); W99: Webb et al. (1999).

2007), making it a triple star system (Feigelson et al. 2006). It was consequently observed less often as it was considered a less likely planet host. We also note that of the 20 young stars in this sample, 14 of them (70\%) are members of wide binary star systems, with separations ranging from 29 to 1967 AU. These include AG Tri A and B, GJ 799 A and B, GJ 871.1 A and B, GJ3305, TWA 8A and 8B, TWA 9A and 9B, TWA 11B, TWA $13 \mathrm{~A}$ and $13 \mathrm{~B}$.

In addition to these 20 young stars, we also included in our sample the well-studied single M dwarf flare star GJ 873 (EV Lac). GJ 873 is the second brightest $\mathrm{M}$ dwarf $\mathrm{X}$-ray source in the Röntgensatellit All-Sky Survey (Hünsch et al. 1999; Osten et al. 2006), has one of the strongest surface magnetic field strengths measured on an M dwarf (Johns-Krull \& Valenti 1996; Reiners \& Basri 2007), and a differential star spot covering factor of 4\%-11\% (Abranin et al. 1998). While its activity suggests a young age, its dwarf-like gravity and the absence of lithium in its atmosphere (Pettersen et al. 1984) indicate that it is not premain sequence. It is usually classified as a young disk star (e.g., Osten et al. 2006) with an approximate age of $10^{8} \mathrm{yr}$. The motivation for including this star was to assess the achievable $\mathrm{RV}$ precision at infrared wavelengths in the limit of extreme surface activity.

For calibration purposes, we observed a handful of A spectraltype stars during each observing run. The telluric absorption fea- tures superimposed upon these nearly featureless stellar spectra, at infrared wavelengths, are used to characterize the instrumental profile. In addition, we observed three mid-M dwarf stars (GJ 628, GJ725A, and GJ725B) that have been previously monitored with high-precision optical RV techniques and shown to have no detectable RV companions (Nidever et al. 2002; Endl et al. 2006); Endl et al. (2006) measure $\mathrm{RV}$ dispersions of $7.4 \mathrm{~m} \mathrm{~s}^{-1}$ and $7.1 \mathrm{~m} \mathrm{~s}^{-1}$ for GJ $725 \mathrm{~A}$ and GJ $725 \mathrm{~B}$, respectively. These stars were included to assess the achievable RV precision in the limit of slowly rotating, inactive stars.

Stellar masses for the young stars are estimated by comparing their stellar temperatures to a theoretical isochrone. To do this, we assembled stellar spectral types from original references (see Table 1) and assigned temperatures using the T Tauri like temperature scale of Luhman (2003) for stars cooler than M0, and the dwarf temperature scale of Kraus \& Hillenbrand (2007) for hotter stars. These temperatures are then compared to the 10 Myr isochrone of Baraffe et al. (1998) with a mixing length of 1.9 above $0.6 M_{\odot}$ and a mixing length of 1.0 at lower masses; this evolutionary model with the adopted temperature scale is consistent with the available dynamical mass constraints for young stars (Hillenbrand \& White 2004; Mathieu et al. 2007). This yields stellar masses that range from $0.12 M_{\odot}$ to $1.01 M_{\odot}$, with a median mass of $0.6 M_{\odot}$. Approximate masses for the 
field dwarfs are estimated from the absolute magnitude-mass relations of Henry \& McCarthy (1993), where the absolute magnitudes have been assigned based on spectral-type relations given in Kraus \& Hillenbrand (2007).

Table 1 summarizes the basic properties of the sample observed, excluding the A spectral-type stars. The listed properties include spectral types, $K$ magnitudes, mass estimates, rotational periods, if known, previously reported $v \sin i$ values, if available, and the corresponding references. The $K$ magnitudes for the field stars are taken from Leggett (1992) whereas the values for Association members are from the Two Micron All Sky Survey (2MASS) survey, and thus are $K_{s}$ magnitudes. Since the 2MASS survey reports only a combined magnitude for GJ799AB, a 2".8 pair, we estimate each component's magnitude by assuming that they are equally bright; the components of this binary have the same spectral type.

\section{HIGH-DISPERSION INFRARED SPECTRA}

Spectroscopic observations of the sample stars were obtained using the cross-dispersed infrared echelle spectrograph NIRSPEC (McLean et al. 1998) on the W. M. Keck II telescope. The observations were obtained over 12 observing runs in total, spanning from 2004 November 16 to 2009 May 12. The large number of runs was a consequence of this program being completed in concert with a similar program focused on L dwarfs (Blake et al. 2007, 2010).

All observations were obtained with the three pixel (0.'432) slit in combination with the N7 blocking filter, and approximate echelle angle 62.65 and grating angle 35.50. This yielded seven orders of spectra spanning approximately $1.99-2.39 \mu \mathrm{m}$, with gaps between the orders, at a resolving power of approximately 30,000 (measured as described below). The spectra were obtained in pairs at two locations along the slit. Collecting observations in this manner provided a nearly simultaneous measurement of sky emission and detector bias for both images.

Since the measurement goal of this program was precise RVs, considerable effort was put into maintaining instrument stability by not moving any internal components of NIRSPEC while observing. However, when the NIRSPEC software is first started (at the beginning of a night) or when a software crash occurs, the motors for the filter wheels and reflection angles must all be reinitialized, forcing the internal components to move. Since resetting the reflection angles typically results in a slightly different setting, these values were tweaked slightly, by eye, to return to the primary wavelength setting; this procedure resulted in the same wavelength coverage to within a few pixels. Since the spectroscopic properties of this new setting would be slightly different, we strived to characterize each "observational set" by intensively observing one of the A spectral-type stars for each NIRSPEC setting. We were successful in this effort for all but one "observational set."

\section{IMAGE REDUCTION AND SPECTRAL EXTRACTION}

For each observational set, described above, dark images were median combined and this median was subtracted from each flat-field image. These subtracted flat-field images were then normalized by counts in the central $\sim 10 \%$ of the array and then median combined to generate a master flat. Regions outside the illuminated seven orders were set to unity. The orders were not normalized in the direction of dispersion, a necessary step to strictly preserve the counts and the correct relative signal-to-noise ratio $(\mathrm{S} / \mathrm{N})$; skipping this led to fringing effects that were more consistent, smaller in amplitude, and easier to remove (see below). All spectrum images were then divided by the master flat constructed for its observational set, and then each flattened image had its corresponding nod image subtracted from it to remove the sky emission, detector bias, and dark current. This yielded two reduced spectrum images for each epoch.

Prior to spectral extraction, the seven spectral orders were located on the reduced spectrum image automatically by finding maxima in smoothed spatial profiles of each order (i.e., perpendicular to the dispersion direction). Gaussian functions were then fit to these spatial profiles in binned segments of 10 columns along the orders. The orders were then traced by fitting a low-order polynomial to the peak position of these Gaussians, with $3 \sigma$ outliers excluded from the fit. Next, regions that were more than $3 \sigma$ above or below the best-fit polynomial defining the center of each order were masked and excluded from any further analysis. Masking the orders in this way objectively accounted for variations in the spatial width of the spectra, usually caused by variations in the seeing, and proved vital for successful extraction of pair subtracted spectra only 10.'0 apart.

Spectra were extracted from the masked images using a modified version of the "optimal extraction" procedure described in Horne et al. (1986). Unlike standard extraction techniques which simply sum pixel values over a specified range, optimal extraction sums pixels weighted by the variance of an assumed smoothly varying spatial profile that can be defined either perpendicular to or parallel to the dispersion direction. This minimizes the noisy contributions of profile wings and allows significant deviates from the profile (e.g., hot pixels and cosmic rays) to be easily identified and removed (see Piskunov \& Valenti 2002 for this and other issues related to the optimal extraction of cross-dispersed echelle spectra). After considerable experimentation, we adopted spatial profiles defined parallel to the dispersion direction and used a Gaussian plus second order polynomial to model these. The variance of the profile was determined from each pair subtracted flat-fielded spectrum image. We note that this is slightly different from the recommendation of Horne et al. (1986) to use a bias subtracted flat-fielded spectrum image; as with most infrared observations, the bias is only removed as a part of pair subtraction. The addition of these bias counts produced slightly larger variance estimates, but this yielded no noticeable effect on our relatively high $\mathrm{S} / \mathrm{N}$ spectra. Future studies may consider obtaining bias images throughout the night in an attempt to determine a more accurate variance image.

An example of an optimally extracted spectrum is shown in Figure 1. One feature not accounted for in these extractions is interference fringing. Although some investigators have developed prescriptions to remove this via Fourier filtering (Deming et al. 2005), we accounted for this feature in the spectral modeling process.

\section{MODELING THE SPECTRA AND PRECISE RADIAL VELOCITY MEASUREMENTS}

\subsection{Assumptions}

Precise RVs were extracted from the observations by constructing a detailed model of each spectrum. Although a wide variety of modeling prescriptions were investigated with our data to optimize this, the prescription described here is the one that yielded the most consistent RVs for 15+ epochs of three slowly rotating $\mathrm{M}$ dwarfs with no known planets (GJ 628, GJ725A, and GJ725B). 
Table 2

Survey Results

\begin{tabular}{|c|c|c|c|c|c|c|c|c|c|}
\hline $\begin{array}{l}\text { Star } \\
\text { Name }\end{array}$ & $\begin{array}{c}\text { Model } \\
T_{\text {eff }}, \log (g)\end{array}$ & $\begin{array}{c}v \sin i \\
\left(\mathrm{~km} \mathrm{~s}^{-1}\right)\end{array}$ & $\begin{array}{c}\langle\mathrm{RV}\rangle \\
\left(\mathrm{m} \mathrm{s}^{-1}\right)\end{array}$ & $N$ & $\begin{array}{c}\sigma_{\mathrm{obs}} \\
\left(\mathrm{m} \mathrm{s}^{-1}\right)\end{array}$ & $\begin{array}{c}3 \text { days } \\
\left(M_{J}\right)\end{array}$ & $\begin{array}{c}10 \text { days } \\
\left(M_{J}\right)\end{array}$ & $\begin{array}{c}30 \text { days } \\
\left(M_{J}\right)\end{array}$ & $\begin{array}{c}100 \text { days } \\
\left(M_{J}\right)\end{array}$ \\
\hline \multicolumn{10}{|c|}{ Single field stars } \\
\hline GJ 628 & $3400,4.8$ & $1.7 \pm 0.1$ & $-21113 \pm 15$ & 13 & 55 & 3.7 & 5.3 & 8.7 & 10.5 \\
\hline GJ 725A & $3400,4.8$ & $1.8 \pm 0.1$ & $-611 \pm 12$ & 18 & 51 & 5.0 & 6.9 & 13 & 16 \\
\hline GJ 725B & $3400,4.8$ & $1.8 \pm 0.1$ & $+1321 \pm 13$ & 18 & 53 & 3.7 & 5.9 & 9.2 & 13 \\
\hline \multicolumn{10}{|c|}{ Chromospherically active field stars } \\
\hline GJ 873 & $3400,4.8$ & $4.7 \pm 0.1$ & $+545 \pm 5$ & 20 & 115 & 5.1 & 7.9 & 12 & 16 \\
\hline \multicolumn{10}{|c|}{$\beta$ Pic stars } \\
\hline AU Mic & $3800,4.2$ & $8.7 \pm 0.2$ & $-4130 \pm 33$ & 14 & 125 & 11 & 17 & 23 & 32 \\
\hline AG Tri A & $3800,4.2$ & $4.7 \pm 0.1$ & $+6743 \pm 26$ & 14 & 98 & 10 & 17 & 22 & 33 \\
\hline AG Tri B & $3800,4.2$ & $5.0 \pm 0.1$ & $+5961 \pm 35$ & 14 & 132 & 9.9 & 14 & 20 & 29 \\
\hline GJ 182 & $3800,4.2$ & $9.4 \pm 0.7$ & $+19818 \pm 42$ & 6 & 103 & 12 & 19 & 25 & 54 \\
\hline GJ 3305 & $3800,4.2$ & $5.7 \pm 0.1$ & [+20625] & 5 & 457 & $\ldots$ & $\ldots$ & $\ldots$ & $\ldots$ \\
\hline GJ 799 A & $3800,4.2$ & $9.6 \pm 0.6$ & $-3727 \pm 40$ & 14 & 151 & 3.9 & 5.9 & 8.2 & 12 \\
\hline GJ 799 B & $3200,4.2$ & $14.7 \pm 0.4$ & $-5126 \pm 50$ & 13 & 179 & 6.0 & 7.7 & 12 & 18 \\
\hline GJ $871.1 \mathrm{~A}$ & $3200,4.2$ & $13.9 \pm 0.5$ & $+3087 \pm 36$ & 14 & 134 & 5.7 & 8.6 & 12 & 19 \\
\hline GJ $871.1 \mathrm{~B}$ & $3200,4.2$ & $22.7 \pm 0.3$ & $+2031 \pm 44$ & 15 & 169 & 5.2 & 7.3 & 10 & 15 \\
\hline HIP 12545 & $3800,4.2$ & $8.7 \pm 0.2$ & $+8253 \pm 48$ & 14 & 179 & 12 & 17 & 23 & 33 \\
\hline \multicolumn{10}{|c|}{ TW Hya stars } \\
\hline TWA 7 & $3800,4.2$ & $4.7 \pm 0.1$ & $+12446 \pm 14$ & 12 & 94 & 7.4 & 12 & 19 & 25 \\
\hline TWA 8A & $3600,4.2$ & $4.7 \pm 0.1$ & $+8679 \pm 23$ & 15 & 90 & 6.7 & 10 & 15 & 22 \\
\hline TWA 8B & $3400,4.2$ & $10.5 \pm 0.3$ & $+8607 \pm 32$ & 15 & 123 & 3.6 & 4.5 & 7.1 & 11 \\
\hline TWA 9A & $4000,4.2$ & $10.4 \pm 0.3$ & $+11649 \pm 21$ & 11 & 71 & 15 & 25 & 30 & 47 \\
\hline TWA 9B & $3800,4.2$ & $8.6 \pm 0.3$ & $+12279 \pm 27$ & 11 & 90 & 8.8 & 15 & 20 & 29 \\
\hline TWA 11B & $3600,4.2$ & $12.0 \pm 0.2$ & $+8923 \pm 59$ & 11 & 191 & 10 & 12 & 19 & 29 \\
\hline TWA 12 & $3600,4.2$ & $17.0 \pm 0.3$ & $+12498 \pm 52$ & 12 & 181 & 11 & 14 & 20 & 31 \\
\hline TWA 13A & $3800,4.2$ & $11.1 \pm 0.3$ & $+11668 \pm 65$ & 14 & 242 & $\ldots$ & $\ldots$ & $\ldots$ & $\ldots$ \\
\hline TWA 13B & $3600,4.2$ & $10.7 \pm 0.3$ & $+12075 \pm 43$ & 13 & 149 & 9.0 & 13 & 18 & 26 \\
\hline TWA 23 & $3800,4.2$ & $20.5 \pm 5.5$ & {$[+6520]$} & 15 & 2425 & $\ldots$ & $\ldots$ & $\ldots$ & $\cdots$ \\
\hline
\end{tabular}

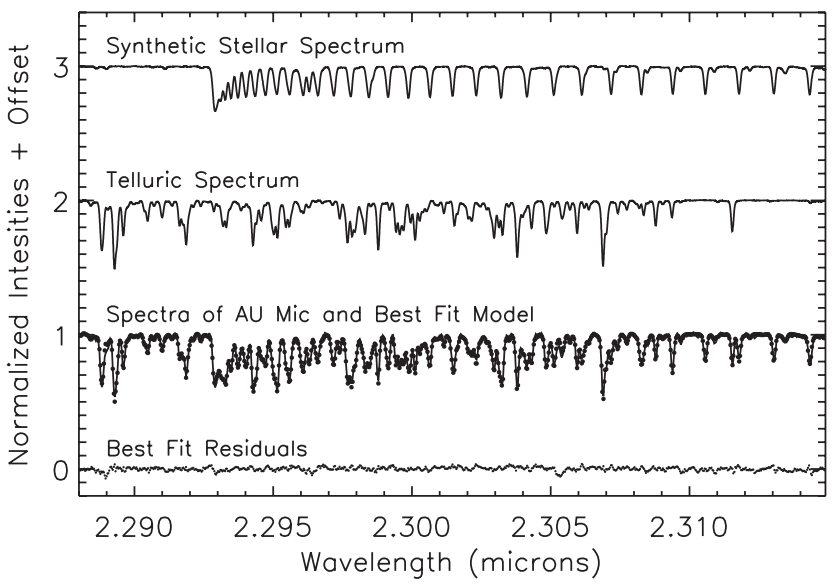

Figure 1. Illustration of the procedure to derive precise radial velocities using NIRSPEC data. A synthetic spectrum (top spectrum) is combined with a telluric stellar spectrum (second spectrum) to construct a model spectrum (third spectrum, dotted line; difficult to see) that is optimized to match an observed spectrum of AU Mic (third spectrum, solid line). The normalized dispersion of the best-fit residuals (fourth spectrum) is $1.5 \%$, and is characteristic of the majority of our fits.

The modeled spectra were constructed from an ultra-high resolution KPNO/Fourier transform spectroscope telluric spectrum, extracted from observations of the Sun (Livingston \& Wallace 1991), and synthetically generated stellar spectra, computed from updated and improved NextGen models (Hauschildt et al. 1999), constructed and provided by coauthor T. Barman. Although the ${ }^{12} \mathrm{CO}$ band head and R-branch lines are tempera- ture and surface gravity dependent, we did not determine these parameters from our spectra; attempts to do this yielded results that were only marginally consistent with previous values considered to be more accurate (e.g., from optical spectra). Instead, we used a synthetically generated spectrum that most closely matched each star's spectral type and expected surface gravity. All field stars (GJ 628, GJ725A, GJ725B, GJ 873) were assumed to have a $\log (g)=4.8 \mathrm{dex}$, while the young stars were assumed to have a $\log (g)=4.2$ dex, consistent with recent $\log (g)$ measurements (Mentuch et al. 2008). The stellar properties of the spectral templates used for each star are listed in Table 2.

Each observation was modeled by combining a telluric and a synthetic spectrum, parameterized by 10 free parameters. Four parameters determine instrumental properties - the wavelength solution was parameterized by a quadratic polynomial, characteristic of slightly curved spectral orders, and the instrumental spectral profile was modeled as a single best-fit Gaussian for the entire order. The remaining six parameters characterize the spectral properties, including the depth of telluric features, the depth of stellar features, the star's projected rotational velocity $(v \sin i)$, a linear (two parameter) continuum normalization offset, and finally, the star's RV. The limb darkening coefficient used to calculate rotationally broadened profiles, which was not considered a free parameter in these calculations, was held fixed at 0.6 in all cases.

\subsection{Implementation}

Only one of the seven orders acquired (NIRSPEC order 33) was modeled; this is one of the few wavelength regions which 
has a sufficiently rich array of both the stellar and telluric absorption features, permitting precise RV calibration. The usable region of the observed order spans approximately $270 \AA$, from $2.288 \mu \mathrm{m}$ to $3.315 \mu \mathrm{m}$ (Figure 1 ).

The spectral modeling proceeded in essentially three stages. First, the A-type star spectra were modeled to construct initial estimates of the wavelength solution and instrumental profile for each observational set. Since the spectra of A-type stars are essentially featureless over this wavelength range, their spectra were modeled as simple telluric spectra, and 3 of the 10 parameters of the general fit were omitted (stellar absorption depth, $v \sin i$, and RV). The best fit was determined by subtracting the model spectrum from the observed spectrum and applying a Hamming filter to this difference to remove the residual fringing pattern. Optimization was achieved by an iterative process that minimized the variance-weighted reduced $\chi^{2}$ of these filtered residuals, using the prescription described below. Parameters determined for multiple A star observations within a set were averaged, weighted by their $\chi^{2}$ values, to determine the best "first guess" values for that set.

The average instrumental profile determined from the best-fit models of the A star spectra corresponded to a resolving power of $\sim 30,000$, with a dispersion of $\sim 2000$. This is larger than expected for this instrumental setup, which is predicted to yield resolving powers of $\sim 25,000$ (McLean et al. 1998). Although we are unsure of the discrepancy, it may be attributable to the excellent seeing at Mauna Kea and starlight not fully filling the 0.432 slit.

Each late-type stellar spectrum was modeled by adopting and holding fixed its set's estimates of the linear and quadratic terms of the wavelength solution. All other parameters were determined via an iterative fitting process, but at all times the parameters were nevertheless restricted to realistic ranges. In the first iteration the zeroth-order component of the wavelength solution was optimized by allowing it to vary; the parameter describing the instrument profile was adopted from its set's estimate. The second iteration optimized the instrument profile by allowing it to vary. The third iteration was used to determine the stellar $v \sin i$ by allowing it to vary. Once complete, a chisquare weighted mean of the best-fit $v \sin i$ value was computed and adopted for the star. In the final iteration only the five remaining parameters (two normalization parameters, telluric depths, stellar depths, RV) were allowed to vary.

For the one observational set without any A star observations (Section 3), the target stars of that set were modeled using the average A star parameters from all other sets as a first guess. The analysis then proceeded as described above. The agreement of measured stellar properties (e.g., RVs, $v \sin i$ ) from this set and other sets suggested the lack of A stars did not compromise the analysis in this case, likely because of the nearly identical observing setups carefully determined each time.

The specific prescription used to determine multipleparameter best fits can have a significant impact on the results. In this first study we adopted a minimization of the filtered difference spectra based on the downhill simplex method of Nelder \& Mead (1965), as implemented by Press et al. (1992). ${ }^{8}$ Our implementation of this prescription enforced user-specified limits on each parameter by restarting an iteration with new parameters if any were out of bounds. The minimization proceeded until

\footnotetext{
8 The version of this multiple-parameter optimization available in the IDL Astronomy User's Library, called $A M E O B A$, must be converted to double-precision to converge self-consistently.
}

both the best-fit $\chi^{2}$ dropped by less than $1 \%$ and all parameters remain consistent with recent values.

Figure 1 shows an example model spectrum for comparison with the observed spectrum of the young star AU Mic. The residuals to the fit have a normalized dispersion of $1.5 \%$, which is typical for the majority of modeled spectra. We note that the results from this last stage yield absolute RVs, since they are determined relative to at rest telluric absorption features. These values are then converted to barycentric velocities using a correction prescription accurate to $\sim 1 \mathrm{~m} \mathrm{~s}^{-1}$ (G. Basri 2011, private communication). For the interested reader, we note that Seifahrt et al. (2008), Blake et al. (2010), Bean et al. (2010), Crockett et al. (2011), and Muirhead et al. (2011) provide detailed descriptions of complementary methods to determine precise RVs with nod-subtracted infrared spectra.

\section{RESULTS}

In Table 2, we summarize the results of our spectroscopic modeling effort to extract RVs. The second column lists the assigned model parameters $\left(T_{\text {eff }}, \log (g)\right)$ and the third column lists the determined $v \sin i$ values. Uncertainties in the $v \sin i$ values are set to be the standard deviation of the $v \sin i$ estimates using all epochs for each star and thus are not necessarily free of systematic effect. The very large uncertainty for TWA 23 may be a consequence of its spectroscopic companion (Section 6.2) broadening its line profiles in some epochs.

The fourth column in Table 2 lists the mean RV and the uncertainty in this mean $(\sigma / \sqrt{N})$, calculated from all observations. The uncertainties in the mean range from $12 \mathrm{~m} \mathrm{~s}^{-1}$ to $65 \mathrm{~m} \mathrm{~s}^{-1}$ for single stars; these are some of the most precise absolute system RV measurements ever for young stars. The fifth and sixth columns list the number of observations $(N)$ and the standard deviation $(\sigma)$ of the RV measurements extracted from these observations. In the following analysis, we include GJ 873 in the statistics determined for young stars.

In the Appendix, RV measurements from each epoch for all stars are provided. The calculation of the measurement uncertainties are describe below. Figure 2 illustrates several RV curves, including the field star GJ725 A (M3.5), the flare star GJ 873 (M3.5), the debris disk host AU Mic, and the components of the wide binary TWA 8 .

In the following sections, the ensemble RV dispersions, as measured by the standard deviations, are used to assess empirically the precision with which RVs can be measured and to identify RV variables.

\subsection{Error Analysis}

For single stars, the RV dispersions are assumed to stem from three causes of variability: (1) a theoretical photon noise error $\left(\sigma_{\text {phot }}\right)$ based on the $\mathrm{S} / \mathrm{N}$ of the observed spectrum and the number and shape of the features in best-fit synthetic and telluric spectra, $(2)$ an instrumental error $\left(\sigma_{\text {inst }}\right)$ based on the characteristics of NIRSPEC spectra, and (3) an intrinsic stellar error $\left(\sigma_{\text {stel }}\right)$ caused by stellar activity. Although the second and third causes are likely attributable to non-stochastic events (e.g., variable bias, fringing or stellar flares, star spots, respectively), we nevertheless assume that these variability sources add in quadrature to produce the observed dispersions $\left(\sigma_{\text {obs }}^{2}=\sigma_{\text {phot }}^{2}+\sigma_{\text {inst }}^{2}+\sigma_{\text {stel }}^{2}\right)$.

Observations of the three slowly rotating single field stars are used to quantify the magnitude of $\sigma_{\text {inst }}$. As noted in Section 2, previous precise RV measurements of these stars show that their 


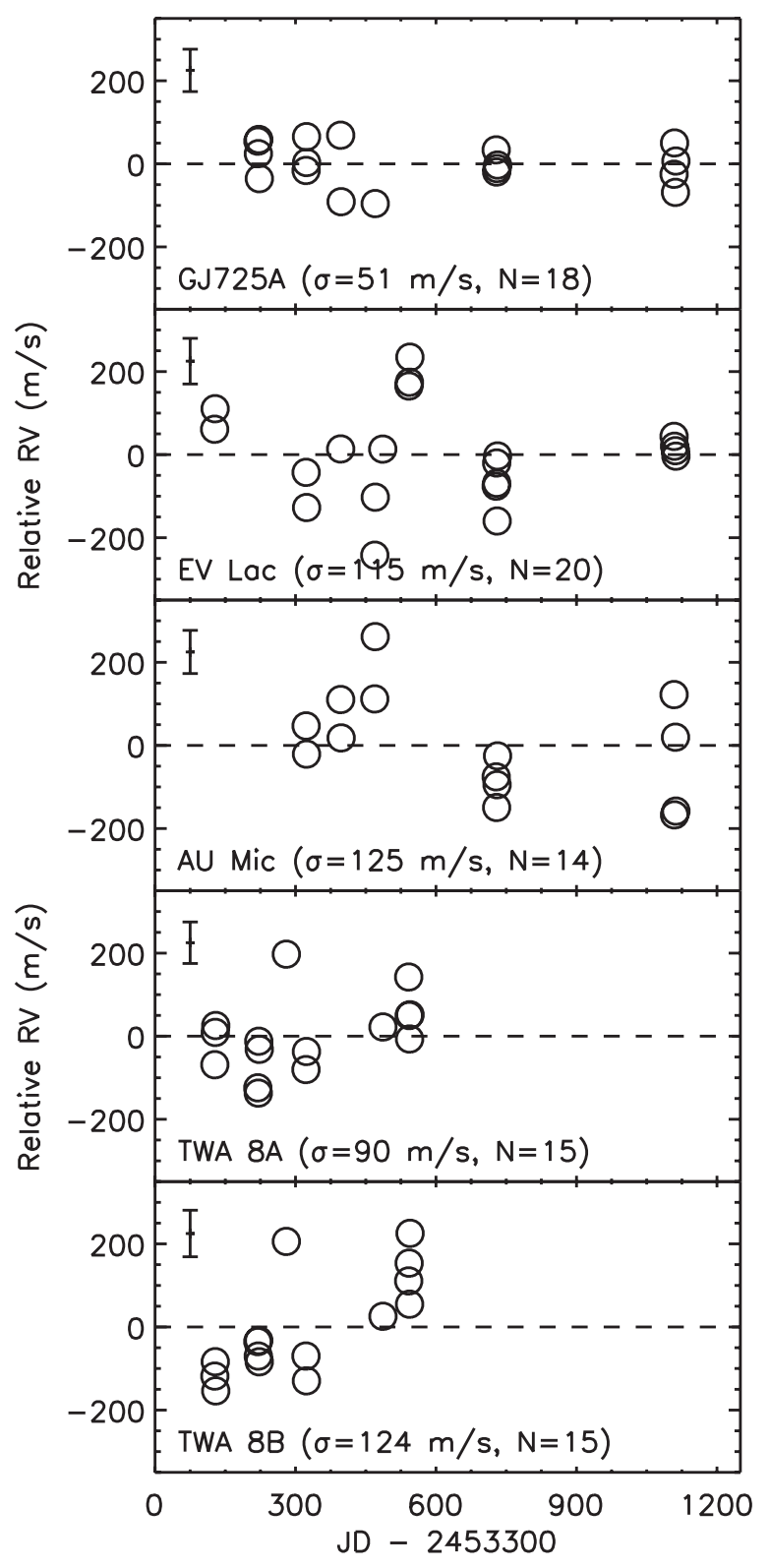

Figure 2. RV curves for the slowly rotating single field star GJ725 A, the chromospherically active star GJ 873, the debris disk host AU Mic, and the components of the wide binary TWA 8; average error bars are illustrated in the upper left of each panel. The dispersions of the field stars, like GJ $725 \mathrm{~A}$, demonstrate an observing precision of $\sim 50 \mathrm{~m} \mathrm{~s}^{-1}$ using high $\mathrm{S} / \mathrm{N}$ spectra of slowly rotating inactive stars. The larger dispersions of other stars are primarily a consequence of enhanced stellar activity.

$\sigma_{\text {stel }}$ is small $\left(<10 \mathrm{~m} \mathrm{~s}^{-1}\right)$; we assign values of zero for all three stars. The $\sigma_{\text {phot }}$ values are calculated for each pair-averaged observation using the $\mathrm{S} / \mathrm{N}$ and a prescription similar to that outlined in Butler et al. (1996). Essentially, this error term is computed as the photon-limited Doppler error of the best-fit synthetic spectrum added in quadrature with the photon-limited wavelength error of the best-fit telluric spectrum. Because we successfully achieved similar high $\mathrm{S} / \mathrm{Ns}$ for all observations, the median pair-averaged $\sigma_{\text {phot }}$ for the three field stars are similar $\left(26.2 \mathrm{~m} \mathrm{~s}^{-1}, 26.4 \mathrm{~m} \mathrm{~s}^{-1}, 27.1 \mathrm{~m} \mathrm{~s}^{-1}\right)$. We use these to estimate $\sigma_{\text {inst }}$ by removing the photon noise error $\sigma_{\text {phot }}$ from the observed dispersion values $\left(\sigma_{\text {inst }}^{2}=\sigma_{\text {obs }}^{2}-\sigma_{\text {phot }}^{2}\right)$. The $\sigma_{\text {inst }}$ values for these three stars are consistent to within $4 \mathrm{~m} \mathrm{~s}^{-1}$, with a median of $46 \mathrm{~m} \mathrm{~s}^{-1}$. These values are only slightly

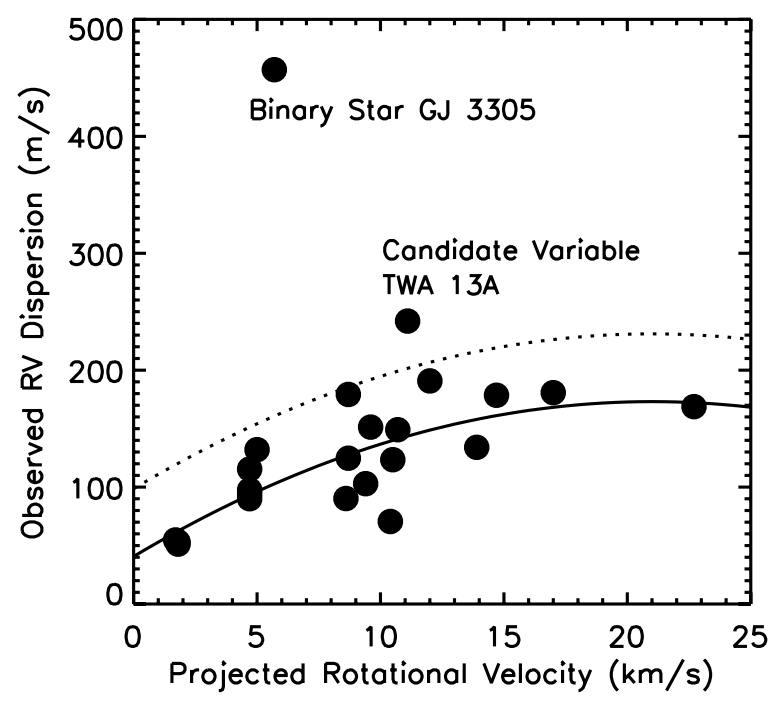

Figure 3. RV dispersion vs. projected rotational velocities; with a dispersion of $2425 \mathrm{~m} \mathrm{~s}^{-1}$, TWA 23 is not illustrated on this plot. A quadratic function is fitted via least squares to the data (solid line) and systems with dispersions $2 \sigma$ above this best fit (dotted line) are considered candidate RV variables.

smaller than their observed dispersions, as expected for high $\mathrm{S} / \mathrm{N}$ spectra of inactive stars. We note that a possible error term not accounted for in these calculations is the stability of the telluric features used for calibration. However, optical (Cochran et al. 1988) and infrared (Seifahrt et al. 2010) RV studies conducted with other facilities have shown these features to be stable to $\lesssim 10 \mathrm{~m} \mathrm{~s}^{-1}$; their stability is thus not a limiting factor for precisely measured RVs with NIRSPEC. These results imply that achieving RV precisions significantly better than $\sim 40 \mathrm{~m} \mathrm{~s}^{-1}$ with NIRSPEC will be challenging unless some methods are developed to account for the instrumental noise, which we presume is attributable to the features of its 1990 s generation array (McLean et al. 1998, e.g., read noise, fringing). It is also possible that some improvements may be realized using alternative spectral modeling techniques that incorporate template spectra instead of synthetic spectra and/or multipleGaussian instrumental profiles. Errors inherent to our modeling prescription are incorporated into the instrument error reported here. Regardless, this precision is considerably better than intended in the design specifications and a credit to the talent of the instrument team.

Measurement errors are assigned to each observation (listed in the Appendix) by calculating $\sigma_{\text {phot }}$ for each pair-averaged observation, and adding that in quadrature with an assumed constant $\sigma_{\text {inst }}$ of $46 \mathrm{~m} \mathrm{~s}^{-1}$. These errors do not account for any possible errors due to stellar activity $\left(\sigma_{\text {stel }}\right)$. The RV errors listed in the Appendix for the 21 young stars range from $46 \mathrm{~m} \mathrm{~s}^{-1}$ to $79 \mathrm{~m} \mathrm{~s}^{-1}$ with a median values of $53 \mathrm{~m} \mathrm{~s}^{-1}$. The $\sigma_{\text {obs }}$ values are nevertheless significantly larger than these error estimates, ranging from 71 to $197 \mathrm{~m} \mathrm{~s}^{-1}$ for the single stars (identified below). This implies a significant contribution from the $\sigma_{\text {stel }}$ to the observed dispersions.

Since stellar activity is known to be correlated with rotational velocity (e.g., Baliunas et al. 1995), we investigate the relation between the observed dispersions and projected rotational velocities in Figure 3. Although there is considerable spread in the dispersion values near a $v \sin i=10 \mathrm{~km} \mathrm{~s}^{-1}$, the two quantities nevertheless appear to be correlated. Stars with $v \sin i$ values less than $12 \mathrm{~km} \mathrm{~s}^{-1}$ have a median dispersion of $115 \mathrm{~m} \mathrm{~s}^{-1}$ 


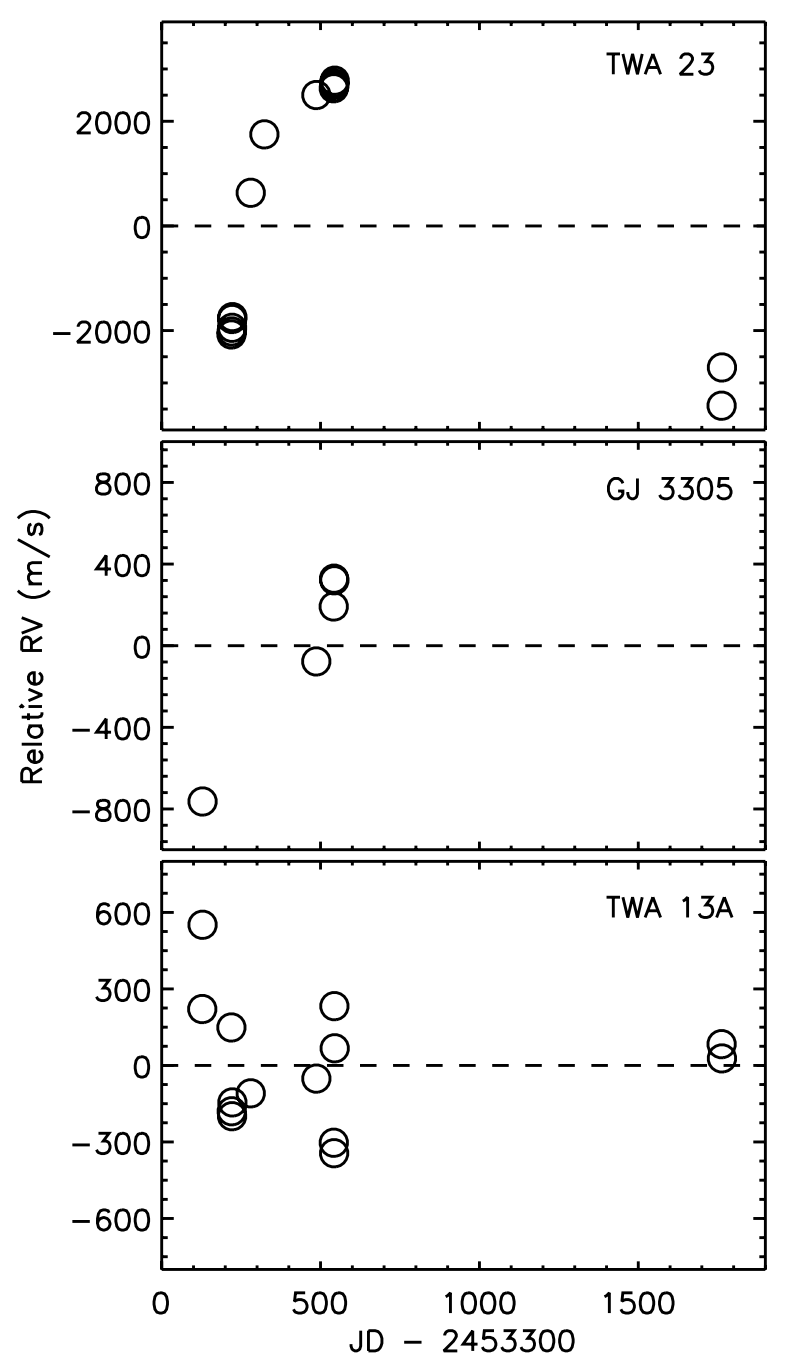

Figure 4. RV curves for the new binary stars TWA 23 and GJ 3305, and the candidate RV variable TWA 13A; typical uncertainties in these measurements are $50-60 \mathrm{~m} \mathrm{~s}^{-1}$. The long timescale (>1 yr) RV trends of TWA 23 and GJ 3305 are indicative of low-mass stellar or possibly brown dwarf companions. In the case of GJ 3305, the motion is likely due to its spatially resolved companion (Kasper et al. 2007). The large amplitude, short-timescale variations of TWA 13A are indicative of a short-period gas giant planet (e.g., a hot Jupiter), but the data are insufficient to confirm this.

while those rotating more rapidly have a median dispersion of $179 \mathrm{~m} \mathrm{~s}^{-1}$.

\subsection{New and Candidate RV Variables}

We use the apparent relation between the observed RV dispersions $\left(\sigma_{\mathrm{obs}}\right)$ and $v \sin i$ values illustrated in Figure 3 to objectively identify possible $\mathrm{RV}$ variables. To do this, a quadratic polynomial is fitted to the data iteratively; stars with dispersions $2 \sigma$ above the best fit are identified as possible RV variables and subsequently excluded from the fit. This identified three stars as possible variable stars, TWA 23, GJ 3305, and TWA 13A. We note that these same stars would be identified using a linear fit to the data, but we adopt and illustrate in Figure 3 a quadratic fit which can account for saturation in the dispersion, if present, given the limited range of dispersions observed (see below).

Two stars have dispersions well above their predicted dispersion for their $v \sin i$, TWA $23\left(\sigma_{\mathrm{obs}}=2425 \mathrm{~m} \mathrm{~s}^{-1}\right)$ and GJ 3305 $\left(\sigma_{\text {obs }}=457 \mathrm{~m} \mathrm{~s}^{-1}\right.$; Table 2$)$. These large dispersions, in combination with their smoothly accelerating RV variations illustrated in

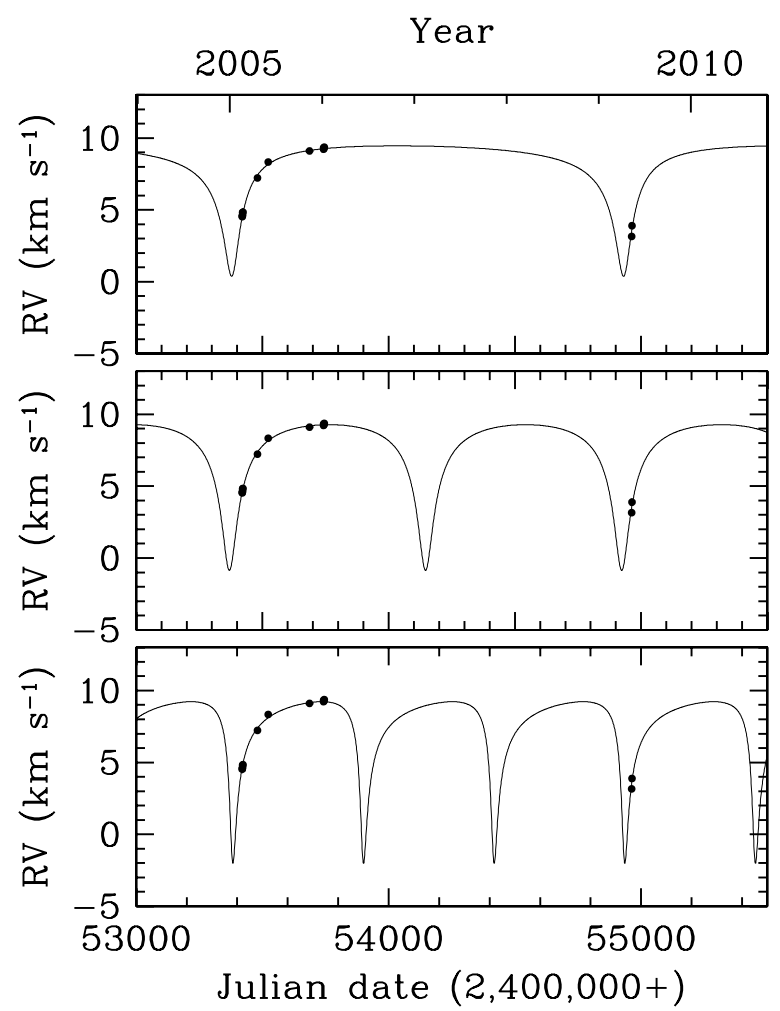

Figure 5. Three plausible orbits for the binary star TWA 23, with periods of 1552 days, 777 days, and 517 days.

Figure 4 confidently indicate that these stars are spectroscopic binaries. In the case of GJ 3305, the observed variations are likely due to its 0 '.093 companion (Kasper et al. 2007). The discovery of this spatially resolved companion midway through our observational program is why we have fewer observations of it; it was considered a less likely place to find a young planet. In the case of TWA 23, the follow-up observations in 2009 helped to establish an upper limit on its orbital period, but its orbit is still underdetermined. In Figure 5 we show three plausible orbital solutions for TWA 23, determined using a standard iterative nonlinear least-squares method (Levenberg-Marquardt, outlined in Press et al. 1992, p. 650); with rms residuals of $\sim 0.2 \mathrm{~km} \mathrm{~s}^{-1}$ in all cases, the three fits are equally good. The orbital periods of these three fits are 517 days, 777 days, and 1552 days with amplitudes of $4 \mathrm{~km} \mathrm{~s}^{-1}, 5 \mathrm{~km} \mathrm{~s}^{-1}$, and $5 \mathrm{~km} \mathrm{~s}^{-1}$. As with GJ 3305, its companion is more likely to have a stellar mass. Nevertheless, the discovery of these relatively low-amplitude spectroscopic binaries highlights the potential for new discoveries that high-precision infrared RVs have when applied to young stars.

The RV dispersion of one other star stands out as being marginally above $(3.4 \sigma)$ the best-fit relation, TWA 13A. Inspection of its RV curve shown in Figure 4 reveals large amplitude variations $\left(>200 \mathrm{~m} \mathrm{~s}^{-1}\right)$ on relatively short (1-2 day) timescales. These types of variations are the type expected if TWA 13A is orbited by a hot Jupiter-like companion. Unfortunately, our sampling and precision are insufficient to confirm this possibility. We classify it as a candidate variable. ${ }^{9}$

\footnotetext{
9 Prior to the follow-up observations in 2009, TWA 13A was even further above the best-fit relation $\left(\sigma_{\mathrm{obs}}=261 \mathrm{~m} \mathrm{~s}^{-1}\right.$ versus $\left.242 \mathrm{~m} \mathrm{~s}^{-1}\right)$; the new observations near its mean reduced its overall dispersion. However, since it was initially identified as a candidate variable for follow-up observations, and remains somewhat discrepant with its expected precision, we continue to classify it as a candidate RV variable.
} 
It is possible that the larger-than-expected RV dispersion of TWA $13 \mathrm{~A}$ is caused by either instrumental errors or stellar activity being larger than expected for this star during these observations; these sources of variation are harder to predict and account for. As a possible test for the former, we compared the RV dispersion of TWA 13A to that of its companion TWA 13B. Both stars are equally bright $(K=7.5)$ and in 13 of TWA 13A's 14 observations, both stars were observed either immediately before or after one another. If the NIRSPEC detector was especially noisy during these observations, it likely would have affected observations of TWA 13B as well. However, the observed dispersion of TWA 13B $\left(\sigma_{\mathrm{obs}}=149 \mathrm{~m} \mathrm{~s}^{-1}\right)$ agrees almost exactly with that predicted from its $v \sin i\left(142 \mathrm{~m} \mathrm{~s}^{-1}\right)$. This strengthens the case that TWA 13A is an RV variable star, but unfortunately it is not yet possible to determine if the variations are caused by a companion or stellar activity.

\section{DISCUSSION}

\subsection{Companion Detection Limits}

Companion mass detection limits are determined via Monte Carlo simulations. Since this survey is most sensitive to shortperiod companions, we set limits only at representative orbital periods, namely 3, 10, 30, and 100 days. The shortest period is chosen to be near the peak in the orbital period distribution of hot Jupiters, while the other periods are chosen to demonstrate how the sensitivity declines with increasing orbital period, given the precision and temporal sampling of this survey. For each star, a set of 10,000 orbits is generated with random inclinations, phases, and companions spanning a broad range of planetary and brown dwarf masses. For discussion purposes, we refer to companions with masses $\leqslant 13 M_{\text {Jupiter }}$ as planets and more massive companions (up to $\sim 65 M_{\text {Jupiter }}$ ) as brown dwarfs. All orbits are assumed to be circular, as would be expected if these companions migrated to these locations via disk interactions (e.g., Lin et al. 1996). In combination with the stellar masses listed in Table 1, these orbits are used to predict a set of RV measurements with the same temporal sampling as each star's set of observations. An assumed Gaussian error of $53 \mathrm{~m} \mathrm{~s}^{-1}$, equal to the median observational error above $\left(\sigma_{\text {phot }} 2+\sigma_{\text {inst }} 2\right)$, is added to these measurements; we note, however, that adding these relatively small errors has no significant effect upon the results. Conservative detection limits are then set by determining the companion mass that induces an RV dispersion that is $2 \sigma$ greater than the expected dispersion for the star's $v \sin i$ (see Figure 3) 99\% of the time; this is consistent with the criteria used to identify candidate variables such as TWA 13A.

The companion detection limits determined from this prescription at 3,10, 30, and 100 days are listed for all single stars in Table 2. The three field stars, which have the smallest RV dispersions, have the lowest companion mass detection limits. On average, these are 4.1, 6.0, 10, and $13 M_{\text {Jupiter }}$ for the above four orbital periods, respectively. These observations confirm that these stars have no short-period massive planet or brown dwarf companions at any orbital orientation except possibly nearly face-on.

In assessing the young stars detection limits, we exclude the known or candidate RV variables and use only results for stars with 11 or more observations (thus excluding GJ 182 with only 6 observations). For this 18 star subsample, the average detection limits are $8.5,13,17$, and $26 M_{\text {Jupiter }}$ at the above four orbital periods, respectively. The lowest mass stars within this subset have the lowest companion detection limits, as

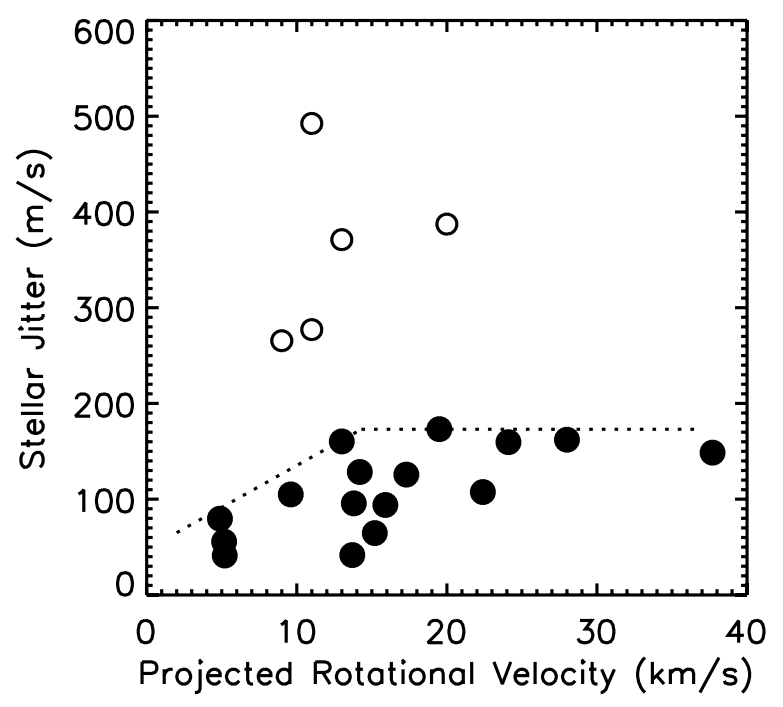

Figure 6. Stellar jitter vs. projected rotational velocity. The stellar jitter at infrared wavelengths (solid circles) is significantly less than at optical wavelengths (empty circles), as expected if it is primarily caused by cool star spots. The dotted line illustrates the upper boundary of infrared stellar jitter values, and illustrates the tentative evidence for saturation at $\sim 180 \mathrm{~m} \mathrm{~s}^{-1}$ for $v \sin i$ values above $12 \mathrm{~km} \mathrm{~s}^{-1}$.

expected since the detection limit scales as $M_{\mathrm{star}}^{2 / 3}$. For example, the $0.12 M_{\odot}$ star TWA $8 \mathrm{~B}$ has the smallest detection limits of $3.6,4.5,7.1$, and $10.6 M_{\text {Jupiter }}$, respectively, despite having an observed dispersion of $123 \mathrm{~m} \mathrm{~s}^{-1}$. Overall, the observations only exclude the presence of the very massive hot $(P<10$ days $)$ Jupiters. However, the observations confidently exclude all hot brown dwarf companions and all but the lowest mass "warm" $(10<P<100 \mathrm{~d})$ brown dwarf companions.

The RV measurements would translate to much stricter companion detection limits if the companion's orbital plane is assumed to be edge-on. This assumption is plausible for the young star AU Mic that is known to harbor an edge-on circumstellar disk (e.g., Kalas et al. 2004). At an assumed age of $12 \mathrm{Myr}$, its M0 spectral type corresponds to a stellar mass of $0.73 M_{\odot}$. For this star, we calculate companion detection limits using the same Monte Carlo simulations described above, but assuming an edge-on orientation for the orbit. This yields companion detection limits of $1.8,2.5,3.9$, and $5.2 M_{\text {Jupiter }}$ at the above four periods, respectively. These detection limits are consistent with the lack of any transiting Jupiter-sized planets found in this system (Hebb et al. 2007). If massive Jupiters do exist within the AU Mic system, they have not migrated inward in its $\sim 12$ Myr lifetime.

\subsection{The Effect of Stellar Activity on Precision RV Measurements}

One of the primary motivations for conducting this RV survey of young stars at infrared wavelengths, as opposed to optical wavelengths, was to mitigate the RV variations induced by stellar activity; we subsequently refer to this as stellar jitter. To investigate the success of this, we compare estimates of the stellar jitter of our infrared observations to the estimates from optical observations. The infrared stellar jitter is estimated by subtracting, in quadrature, the instrumental and average theoretical errors from the observed dispersion for each star $\left(\sigma_{\text {stel }}^{2}=\sigma_{\text {obs }}^{2}-\sigma_{\text {inst }}^{2}-\sigma_{\text {phot }}^{2}\right)$. This yielded values ranging from $36 \mathrm{~m} \mathrm{~s}^{-1}$ to $181 \mathrm{~m} \mathrm{~s}^{-1}$, with a median value of $113 \mathrm{~m} \mathrm{~s}^{-1}$; these are illustrated in Figure 6 versus $v \sin i$. 
The values are then compared to the stellar jitter values calculated from RV measurements in Paulson \& Yelda (2006), who obtained precise optical RV measurements of six members of the $\beta$ Pic Association, including two stars in the present survey here, AU Mic and GJ 3305. GJ 3305 is not included in these comparisons, however, because we identify it as a spectroscopic binary star. Paulson \& Yelda (2006) obtained precise RVs using the telluric oxygen band at $6900 \AA$ with an instrumental precision of $40 \mathrm{~m} \mathrm{~s}^{-1}$. As with the infrared measurements, the stellar jitter is estimated by subtracting, in quadrature, the theoretical and instrumental uncertainties from the observed velocity dispersions. These results are plotted in Figure 6 versus $v \sin i$, and range from $270 \mathrm{~m} \mathrm{~s}^{-1}$ to $490 \mathrm{~m} \mathrm{~s}^{-1}$, with a mean of $360 \mathrm{~m} \mathrm{~s}^{-1}$. A comparison of these suggests that the stellar jitter at infrared ( $K$ band) is approximately three times less than at optical wavelengths ( $R$ band). This is consistent with the reductions measured by Huélamo et al. (2008) for the similar age star TW Hya. The recent study by Mahmud et al. (2011) reports similar reductions between infrared and optical jitter for the younger T Tauri stars DN Tau and Hubble I 4, and slightly larger reductions (a factor 4-5) for V827 Tau and V836 Tau.

Although the binary star GJ 3305 is not included in these comparisons, we note that Paulson \& Yelda (2006) do not identify it as a spectroscopic binary, despite having observed it 11 times on four different nights. We attribute this to their limited sampling, its apparently low-amplitude RV variations, and the larger stellar jitter associated with optical observations of young stars. We also note that we cannot confirm the claim of Song et al. (2003) that HIP 12545 is a single-lined spectroscopic binary. Although they do not report individual measurements, they claim to have measured large $\left(20 \mathrm{~km} \mathrm{~s}^{-1}\right) \mathrm{RV}$ variations for this star using high-dispersion optical spectra. Our 14 measurements of this star spanning from 2004 November 19 to 2006 July 6 have a dispersion of only $0.18 \mathrm{~km} \mathrm{~s}^{-1}$.

Altogether, these results confirm the expectation that infrared observations mitigate the RV variations due to stellar activity and, moreover, quantify the benefit. As discussed in the introduction, this is expected if the RV variations are primarily caused by cool star spots. However, infrared observations made during other chromospheric events such as giant flares or coronal mass ejections may have little or no benefit over optical measurements. It has been shown that giant flares on active stars can cause temporary shifts in optically determined RV of several hundred $\mathrm{m} \mathrm{s}^{-1}$ (Reiners 2009). Although we do not have information to identify flare or flare-like events in our data, this could explain the occasional large (>200 m s${ }^{-1}$ ), one-time RV changes seen for some stars. One of our clearest examples occurred during observations of the slowly rotating star TWA 7 ( $v \sin i=4.5 \mathrm{~km} \mathrm{~s}^{-1}$ ). As illustrated in Figure 7, all but 1 of its $12 \mathrm{RV}$ measurements are consistent to within $100 \mathrm{~m} \mathrm{~s}^{-1}\left(\sigma_{\mathrm{obs}}=\right.$ $47 \mathrm{~m} \mathrm{~s}^{-1}$ ); the discrepant epoch deviates by $\sim 300 \mathrm{~m} \mathrm{~s}^{-1}$ from the mean RV of the other 11 epochs. Comparisons of stars observed immediately before and after this epoch show no similar large amplitude deviations, which might be expected if the detector was especially noisy at this time; the deviation appears to be limited to this observation. The $\mathrm{S} / \mathrm{N}$ of this observation is also above the mean of the remaining observations, and the spectrum shows no defects, such as an cosmic-ray event that was not corrected in the optimal extraction. This leads us to believe the deviation is intrinsic to the star itself. Because we cannot fully explain the event (or others like it), we highlight it here as a cautionary remark for future surveys. Coordinated photometric and/or spectroscopic observations that target chromospheric

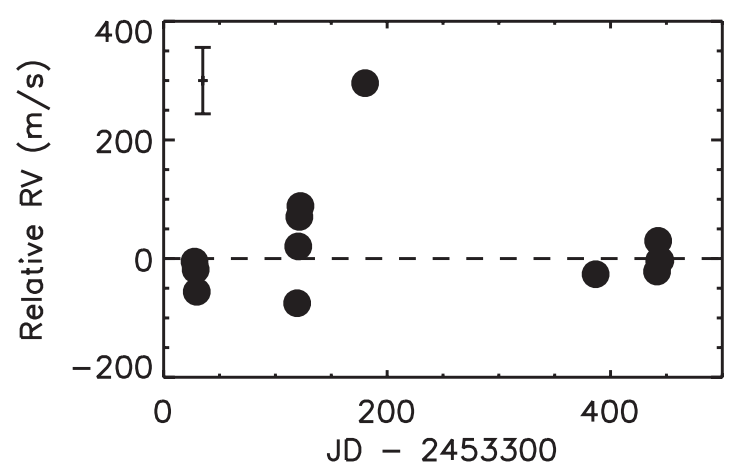

Figure 7. RV curve of the slowly rotating young star TWA 7; an average error bar is illustrated in the upper left. All but 1 of the RVs are consistent to within $100 \mathrm{~m} \mathrm{~s}^{-1}(\sigma=47)$. The one outlier is possibly due to a transient stellar event such as a flare.

indicators may help identify these events, which when excluded, would improve the overall sensitivity to low-mass companions.

Finally, the ensemble infrared stellar jitter results provide a guide for the achievable RV precision for young active stars in the limit of high $\mathrm{S} / \mathrm{N}$ spectra with no wavelength calibration error. For the slowest rotating stars $\left(v \sin i<6 \mathrm{~km} \mathrm{~s}^{-1}\right)$, the median stellar jitter is $77 \mathrm{~m} \mathrm{~s}^{-1}\left(1 \sigma\right.$ spread of $\left.21 \mathrm{~m} \mathrm{~s}^{-1}\right)$. For stars with modest rotation $\left(6 \mathrm{~km} \mathrm{~s}^{-1}<v \sin i<12 \mathrm{~km} \mathrm{~s}^{-1}\right)$, the median stellar jitter is $108 \mathrm{~m} \mathrm{~s}^{-1}\left(1 \sigma\right.$ spread of $\left.43 \mathrm{~m} \mathrm{~s}^{-1}\right)$. And for the most rapidly rotating stars $\left(v \sin i\right.$ of $\left.12-23 \mathrm{~km} \mathrm{~s}^{-1}\right)$, the median stellar jitter is $168 \mathrm{~m} \mathrm{~s}^{-1}\left(1 \sigma\right.$ spread of $\left.24 \mathrm{~m} \mathrm{~s}^{-1}\right)$. We also note that none of the four stars with $v \sin i$ values $>12 \mathrm{~km} \mathrm{~s}^{-1}$ have stellar jitter values above $180 \mathrm{~m} \mathrm{~s}^{-1}$, possibly hinting at stellar jitter saturation (Figure 6). Although the available data cannot robustly confirm this, we highlight this possibility because this $v \sin i$ limit is similar to the observed $\mathrm{X}$-ray saturation limit of $\sim 15 \mathrm{~km} \mathrm{~s}^{-1}$, though for slightly more massive stars in the Pleiades (Stauffer et al. 1994); the $\mathrm{X}$-ray saturation is interpreted as a consequence of maximal coverage of star spots. Likewise, we find that stars with the largest stellar jitter have rotational periods $<3.3$ days (Table 1). As demonstrated in (Pizzolato et al. 2003), subsolar mass stars with rotation periods $<3.0-3.5$ days (depending somewhat on mass) all have saturated X-ray emission. Given that the most rapidly rotating stars studied here appear to be near or beyond the regime where X-rays saturate, its plausible to presume that the stellar jitter is also saturated. The results have implications for how precisely the radial velocities of rapidly rotating stars can be determined.

\section{SUMMARY}

We present the results of a high-precision infrared RV study of 20 young stars in the $\beta$ Pic and TW Hya Associations, as well as the chromospherically active, young disk M3.5 star GJ 873. High spectral resolution $(R \sim 30,000)$ measurements at $2.3 \mu \mathrm{m}$ were obtained with NIRSPEC at the Keck Observatory; the majority of stars have more than a dozen epochs, with a typical temporal sampling of several observations over a week-long run, and then several runs over two to three years. Precise RVs are determined using telluric absorption features as an absolute wavelength reference. Each observation is modeled as the combination of a telluric spectrum and a synthetically generated stellar spectrum, both convolved by a parameterized instrumental profile and projected onto a parameterized wave- 
length solution. The best fit, which includes the RV of the star, is determined by minimizing the $\chi^{2}$.

This modeling technique yields RV dispersions of $\sim 50 \mathrm{~m} \mathrm{~s}^{-1}$ for $13+$ epochs each of three slowly rotating field stars. The dispersion of these RV measurements is dominated by instrumental errors and thus $\sim 50 \mathrm{~m} \mathrm{~s}^{-1}$ can be considered a practical precision limit for NIRPSEC (see also Blake et al. 2010); this precision is nevertheless well above NIRSPEC's original design specifications. Significant improvements to this precision will likely require a new detector to mitigate dominant noise sources such as fringing, variable bias, and read noise.

The observed RV dispersions for young stars range from $48 \mathrm{~m} \mathrm{~s}^{-1}$ to $197 \mathrm{~m} \mathrm{~s}^{-1}$. These dispersions are dominated by noise from stellar activity or stellar jitter. We estimate the contribution from stellar jitter by subtracting, in quadrature, the average instrumental noise $\left(46 \mathrm{~m} \mathrm{~s}^{-1}\right)$ and the calculated theoretical noise $\left(\sim 40 \mathrm{~m} \mathrm{~s}^{-1}\right)$ from the observed dispersions. The stellar jitter increases with projected rotational velocity $(v \sin i)$. The slowest rotating stars $\left(v \sin i<6 \mathrm{~km} \mathrm{~s}^{-1}\right)$ have a median stellar jitter of $77 \mathrm{~m} \mathrm{~s}^{-1}$, modest rotation stars $\left(6 \mathrm{~km} \mathrm{~s}^{-1}<v \sin i<12 \mathrm{~km} \mathrm{~s}^{-1}\right)$ have a median stellar jitter of $108 \mathrm{~m} \mathrm{~s}^{-1}$, and the most rapidly rotating stars $(v \sin i$ of $12-23 \mathrm{~km} \mathrm{~s}^{-1}$ ) have a median stellar jitter of $168 \mathrm{~m} \mathrm{~s}^{-1}$. There is also tentative evidence that stellar jitter saturates at $\sim 180 \mathrm{~m} \mathrm{~s}^{-1}$ above $v \sin i$ values of $\sim 12 \mathrm{~km} \mathrm{~s}^{-1}$, or below rotational periods of $\sim 3-4$ days, consistent with the rate of rotation that causes $\mathrm{X}$-rays to saturate.

These infrared stellar jitter values are, on average, a factor of three less than the stellar jitter values of similar age $\beta$ Pic Association stars observed at optical ( $R$ band) wavelengths. Case studies by Huélamo et al. (2008) and Mahmud et al. (2011) corroborate these reductions. This is expected if stellar jitter is predominantly caused by cool star spots, and confirms that infrared measurements have a significant advantage over optical measurements in obtaining precise RV measurements of active stars.

Three stars have RV dispersions significantly above the level expected for their $v \sin i$. The smoothly accelerating RVs of two of these stars, GJ3305 and TWA 23, indicate that they are single-lined spectroscopic binaries. The RV orbits are still underdetermined. In the case of GJ 3305, the motion is likely caused by its recently identified $\sim 93$ milliarcsec companion (Kasper et al. 2007). The third star, TWA 13A, exhibits large amplitude (>200 $\mathrm{m} \mathrm{s}^{-1}$ ), short-timescale variations indicative of a hot Jupiter-like companion, but the available data are insufficient to confirm this. We label it as a candidate RV variable. We also note that the relative rarity of hot Jupiter companions $(\sim 1 \%)$ implies that a first discovery in this small sample of 20 young stars would be most fortuitous.

For the remainder of the sample, these observations exclude the presence of any "hot" $(P<3$ days $)$ companions more massive than $8 M_{\text {Jup }}$, and any "warm" $(P<30$ day $)$ companions more massive than $17 M_{\text {Jup }}$, on average. Assuming an edge-on orbit for the edge-on disk system AU Mic, these observations exclude the presence of any hot Jupiters more massive than $1.8 M_{\text {Jup }}$ or warm Jupiters more massive than $3.9 M_{\text {Jup }}$. If massive Jupiters exist in the AU Mic system, they have not migrated inward in its $\sim 12$ Myr lifetime.

While the effect of star spots will continue to be a limiting factor in the search for young short-period planets, even at infrared wavelengths, the results presented here elucidate better the opportunities for determining precise RVs from infrared observations in the limit of high stellar activity.
We are grateful to the support staff at Keck Observatory, especially Grant Hill and Jim Lyke, and to the scientists who built and continue to maintain NIRSPEC, especially Dr. Ian McLean. We appreciate the data provided by the NASA/IPAC Infrared Science Archive and the privilege to observe on the revered summit of Mauna Kea. This project was funded by NSF/AAG grant number 0908018.

\section{APPENDIX}

\section{INDIVIDUAL RADIAL VELOCITY MEASUREMENTS}

Below are the individual RV measurements for the 24 stars observed in this survey. The first column provides the star name, the number of RV measurements, the mean RV and its uncertainty, and the standard deviation of RV measurements. The Julian Dates of the observation are listed in the second column and the barycentric RVs and associated uncertainties

Table 3

Observational Sample

\begin{tabular}{|c|c|c|c|}
\hline Star & HJD $-2,400,000$ & $\begin{array}{l}\text { Radial Velocity } \\
\qquad\left(\mathrm{m} \mathrm{s}^{-1}\right)\end{array}$ & $\mathrm{S} / \mathrm{I}$ \\
\hline $\begin{array}{l}\text { GJ } 628 \\
N=13 \\
\overline{\mathrm{RV}}=-21,113 \pm 15 \\
\sigma=54.84\end{array}$ & $\begin{array}{l}53522.337 \\
53523.502 \\
53597.224 \\
53741.688 \\
53742.688 \\
53743.685 \\
53929.374 \\
53930.307 \\
53931.387 \\
54308.353 \\
54309.355 \\
54311.343 \\
54312.274\end{array}$ & $\begin{array}{l}-21,061 \pm 51 \\
-21,077 \pm 52 \\
-21,139 \pm 53 \\
-21,104 \pm 57 \\
-21,140 \pm 52 \\
-21,145 \pm 46 \\
-21,084 \pm 53 \\
-21,028 \pm 53 \\
-21,123 \pm 53 \\
-21,251 \pm 53 \\
-21,145 \pm 49 \\
-21,095 \pm 51 \\
-21,083 \pm 51\end{array}$ & $\begin{array}{l}141 \\
203 \\
183 \\
141 \\
198 \\
264 \\
221 \\
147 \\
205 \\
208 \\
278 \\
214 \\
241\end{array}$ \\
\hline $\begin{array}{l}\text { GJ 725A } \\
N=18 \\
\mathrm{RV}=-611 \pm 12 \\
\sigma=51.24\end{array}$ & $\begin{array}{l}53419.683 \\
53420.687 \\
53421.580 \\
53422.638 \\
53522.547 \\
53523.419 \\
53523.618 \\
53596.363 \\
53597.343 \\
53670.202 \\
53928.535 \\
53929.453 \\
53930.410 \\
53931.463 \\
54308.364 \\
54309.399 \\
54311.377 \\
54312.291 \\
\end{array}$ & $\begin{array}{l}-557 \pm 57 \\
-587 \pm 54 \\
-553 \pm 51 \\
-647 \pm 51 \\
-627 \pm 53 \\
-546 \pm 54 \\
-608 \pm 50 \\
-543 \pm 53 \\
-703 \pm 50 \\
-707 \pm 47 \\
-578 \pm 50 \\
-631 \pm 51 \\
-623 \pm 59 \\
-614 \pm 52 \\
-636 \pm 54 \\
-561 \pm 55 \\
-680 \pm 54 \\
-605 \pm 53 \\
\end{array}$ & $\begin{array}{l}161 \\
186 \\
221 \\
230 \\
187 \\
191 \\
261 \\
191 \\
248 \\
206 \\
275 \\
260 \\
147 \\
173 \\
198 \\
172 \\
176 \\
196\end{array}$ \\
\hline $\begin{array}{l}\text { GJ 725B } \\
N=18 \\
\mathrm{RV}=1,321 \pm 13 \\
\sigma=53.07\end{array}$ & $\begin{array}{l}53419.682 \\
53420.686 \\
53421.578 \\
53422.636 \\
53522.549 \\
53523.421 \\
53523.621 \\
53596.365 \\
53597.344 \\
53670.204 \\
53928.538 \\
53929.455\end{array}$ & $\begin{array}{l}1,305 \pm 55 \\
1,319 \pm 59 \\
1,352 \pm 52 \\
1,329 \pm 50 \\
1,410 \pm 52 \\
1,411 \pm 51 \\
1,329 \pm 52 \\
1,376 \pm 53 \\
1,278 \pm 54 \\
1,388 \pm 47 \\
1,324 \pm 52 \\
1,266 \pm 55\end{array}$ & $\begin{array}{l}178 \\
144 \\
150 \\
261 \\
194 \\
233 \\
233 \\
196 \\
183 \\
151 \\
241 \\
200\end{array}$ \\
\hline
\end{tabular}


Table 3

(Continued)

\begin{tabular}{|c|c|c|c|}
\hline Star & HJD - 2,400,000 & $\begin{array}{l}\text { Radial Velocity } \\
\qquad\left(\mathrm{m} \mathrm{s}^{-1}\right)\end{array}$ & $S / N$ \\
\hline & $\begin{array}{l}53930.412 \\
53931.489 \\
54308.367 \\
54309.401 \\
54311.379 \\
54312.293 \\
\end{array}$ & $\begin{array}{l}1,282 \pm 54 \\
1,347 \pm 53 \\
1,278 \pm 57 \\
1,226 \pm 55 \\
1,253 \pm 53 \\
1,304 \pm 52 \\
\end{array}$ & $\begin{array}{l}194 \\
205 \\
172 \\
172 \\
206 \\
218\end{array}$ \\
\hline $\begin{array}{l}\text { GJ } 182 \\
N=6 \\
\mathrm{RV}=19,818 \pm 42 \\
\sigma=102.91\end{array}$ & $\begin{array}{l}53327.568 \\
53328.596 \\
53686.442 \\
53741.468 \\
53742.409 \\
53743.389 \\
\end{array}$ & $\begin{array}{l}19,753 \pm 53 \\
19,732 \pm 55 \\
19,696 \pm 52 \\
19,941 \pm 50 \\
19,898 \pm 56 \\
19,887 \pm 57\end{array}$ & $\begin{array}{l}172 \\
184 \\
166 \\
224 \\
175 \\
176 \\
\end{array}$ \\
\hline $\begin{array}{l}\text { GJ } 873 \\
N=21 \\
\overline{\mathrm{RV}}=545 \pm 25 \\
\sigma=112.93\end{array}$ & $\begin{array}{l}53327.225 \\
53328.198 \\
53522.592 \\
53523.587 \\
53596.442 \\
53597.401 \\
53669.328 \\
53670.352 \\
53686.247 \\
53742.205 \\
53743.187 \\
53744.187 \\
53928.569 \\
53929.516 \\
53929.615 \\
53930.538 \\
53931.493 \\
54308.411 \\
54309.429 \\
54311.430 \\
54312.359\end{array}$ & $\begin{array}{l}606 \pm 62 \\
656 \pm 47 \\
502 \pm 58 \\
418 \pm 55 \\
559 \pm 55 \\
547 \pm 54 \\
303 \pm 56 \\
442 \pm 54 \\
558 \pm 52 \\
710 \pm 55 \\
719 \pm 58 \\
780 \pm 48 \\
469 \pm 57 \\
525 \pm 51 \\
476 \pm 48 \\
385 \pm 55 \\
542 \pm 49 \\
589 \pm 57 \\
565 \pm 53 \\
554 \pm 57 \\
541 \pm 52\end{array}$ & $\begin{array}{l}142 \\
141 \\
147 \\
136 \\
175 \\
165 \\
136 \\
179 \\
198 \\
167 \\
150 \\
128 \\
179 \\
215 \\
383 \\
170 \\
160 \\
149 \\
152 \\
164 \\
201\end{array}$ \\
\hline $\begin{array}{l}\text { AU Mic } \\
N=14 \\
\mathrm{RV}=-4,130 \pm 33 \\
\sigma=124.74\end{array}$ & $\begin{array}{l}53522.559 \\
53523.549 \\
53596.369 \\
53597.379 \\
53669.193 \\
53670.196 \\
53928.504 \\
53929.448 \\
53930.456 \\
53931.396 \\
54308.426 \\
54309.411 \\
54311.404 \\
54312.356 \\
\end{array}$ & $\begin{array}{l}-4,083 \pm 54 \\
-4,151 \pm 54 \\
-4,020 \pm 52 \\
-4,112 \pm 51 \\
-4,018 \pm 52 \\
-3,869 \pm 47 \\
-4,206 \pm 49 \\
-4,280 \pm 50 \\
-4,225 \pm 54 \\
-4,156 \pm 50 \\
-4,008 \pm 53 \\
-4,298 \pm 50 \\
-4,111 \pm 54 \\
-4,289 \pm 52\end{array}$ & $\begin{array}{l}181 \\
193 \\
206 \\
228 \\
205 \\
212 \\
336 \\
274 \\
187 \\
248 \\
211 \\
284 \\
195 \\
205 \\
\end{array}$ \\
\hline $\begin{array}{l}\text { AG Tri A } \\
N=14 \\
\mathrm{RV}=6,743 \pm 26 \\
\sigma=97.53\end{array}$ & $\begin{array}{l}53327.386 \\
53328.376 \\
53329.442 \\
53421.202 \\
53422.198 \\
53669.334 \\
53670.356 \\
53686.257 \\
53686.512 \\
53742.259 \\
53744.196 \\
53928.604 \\
53929.591 \\
53930.583 \\
\end{array}$ & $\begin{array}{l}6,692 \pm 53 \\
6,650 \pm 57 \\
6,650 \pm 56 \\
6,780 \pm 53 \\
6,766 \pm 53 \\
6,759 \pm 50 \\
6,723 \pm 51 \\
6,552 \pm 53 \\
6,620 \pm 57 \\
6,830 \pm 56 \\
6,878 \pm 48 \\
6,830 \pm 64 \\
6,824 \pm 52 \\
6,840 \pm 52 \\
\end{array}$ & $\begin{array}{l}208 \\
163 \\
151 \\
196 \\
198 \\
228 \\
226 \\
163 \\
140 \\
200 \\
149 \\
123 \\
223 \\
213 \\
\end{array}$ \\
\hline $\begin{array}{l}\text { AG Tri B } \\
N=14\end{array}$ & $\begin{array}{l}53327.389 \\
53328.378\end{array}$ & $\begin{array}{l}5,845 \pm 51 \\
5,939 \pm 53\end{array}$ & $\begin{array}{l}260 \\
212\end{array}$ \\
\hline
\end{tabular}

Table 3

(Continued)

\begin{tabular}{|c|c|c|c|}
\hline Star & HJD - 2,400,000 & $\begin{array}{l}\text { Radial Velocity } \\
\qquad\left(\mathrm{m} \mathrm{s}^{-1}\right)\end{array}$ & $\mathrm{S} / \mathrm{N}$ \\
\hline $\begin{array}{l}\overline{\mathrm{RV}}=5,961 \pm 35 \\
\sigma=132.05\end{array}$ & $\begin{array}{l}53329.445 \\
53421.204 \\
53422.201 \\
53669.336 \\
53670.359 \\
53686.259 \\
53686.514 \\
53742.262 \\
53744.198 \\
53928.609 \\
53929.597 \\
53930.587\end{array}$ & $\begin{array}{l}6,124 \pm 60 \\
6,126 \pm 54 \\
5,920 \pm 52 \\
5,888 \pm 50 \\
5,699 \pm 51 \\
5,871 \pm 52 \\
5,843 \pm 52 \\
6,147 \pm 56 \\
6,073 \pm 48 \\
6,075 \pm 68 \\
5,981 \pm 50 \\
5,923 \pm 52\end{array}$ & $\begin{array}{l}126 \\
193 \\
221 \\
252 \\
231 \\
192 \\
210 \\
240 \\
167 \\
116 \\
271 \\
227\end{array}$ \\
\hline $\begin{array}{l}\text { GJ } 3305 \\
N=5 \\
\mathrm{RV}=20,625 \pm 204 \\
\sigma=457.20\end{array}$ & $\begin{array}{l}53328.592 \\
53686.440 \\
53741.455 \\
53742.407 \\
53743.387\end{array}$ & $\begin{array}{l}19,862 \pm 52 \\
20,548 \pm 58 \\
20,817 \pm 52 \\
20,954 \pm 51 \\
20,946 \pm 52\end{array}$ & $\begin{array}{l}209 \\
159 \\
174 \\
242 \\
237\end{array}$ \\
\hline $\begin{array}{l}\text { GJ } 799 \text { A } \\
N=14 \\
\overline{\mathrm{RV}}=-3,727 \pm 40 \\
\sigma=151.32\end{array}$ & $\begin{array}{l}53522.564 \\
53523.539 \\
53596.372 \\
53597.381 \\
53669.188 \\
53670.193 \\
53928.490 \\
53929.439 \\
53930.450 \\
53931.391 \\
54308.357 \\
54309.406 \\
54311.400 \\
54312.351\end{array}$ & $\begin{array}{l}-3,614 \pm 55 \\
-3,670 \pm 53 \\
-3,902 \pm 49 \\
-4,055 \pm 51 \\
-3,556 \pm 57 \\
-3,572 \pm 47 \\
-3,911 \pm 50 \\
-3,722 \pm 54 \\
-3,658 \pm 52 \\
-3,621 \pm 49 \\
-3,749 \pm 53 \\
-3,850 \pm 54 \\
-3,727 \pm 53 \\
-3,570 \pm 52\end{array}$ & $\begin{array}{l}175 \\
129 \\
273 \\
218 \\
151 \\
204 \\
207 \\
193 \\
164 \\
294 \\
203 \\
185 \\
196 \\
204\end{array}$ \\
\hline $\begin{array}{l}\text { GJ } 799 \mathrm{~B} \\
N=14 \\
\mathrm{RV}=-5,126 \pm 55 \\
\sigma=206.52\end{array}$ & $\begin{array}{l}53522.561 \\
53523.547 \\
53596.374 \\
53597.383 \\
53669.190 \\
53670.190 \\
53928.495 \\
53929.445 \\
53930.454 \\
53931.394 \\
54308.360 \\
54309.408 \\
54311.402 \\
54312.353\end{array}$ & $\begin{array}{l}-4,946 \pm 55 \\
-5,358 \pm 60 \\
-5,165 \pm 53 \\
-4,726 \pm 49 \\
-5,020 \pm 57 \\
-4,888 \pm 49 \\
-4,969 \pm 52 \\
-5,071 \pm 56 \\
-5,310 \pm 59 \\
-5,198 \pm 54 \\
-5,091 \pm 58 \\
-5,326 \pm 55 \\
-5,477 \pm 60 \\
-5,217 \pm 55\end{array}$ & $\begin{array}{l}160 \\
181 \\
253 \\
195 \\
195 \\
173 \\
235 \\
216 \\
181 \\
227 \\
193 \\
217 \\
179 \\
211\end{array}$ \\
\hline $\begin{array}{l}\text { GJ } 871.1 \mathrm{~A} \\
N=14 \\
\mathrm{R} \overline{\mathrm{V}}=3,087 \pm 36 \\
\sigma=134.03\end{array}$ & $\begin{array}{l}53327.203 \\
53328.183 \\
53329.187 \\
53522.582 \\
53523.604 \\
53596.432 \\
53597.394 \\
53669.269 \\
53686.232 \\
53928.518 \\
53929.523 \\
53930.529 \\
54311.415 \\
54312.414\end{array}$ & $\begin{array}{l}3,025 \pm 80 \\
3,082 \pm 55 \\
3,185 \pm 55 \\
3,036 \pm 51 \\
2,831 \pm 60 \\
3,099 \pm 51 \\
3,270 \pm 55 \\
3,054 \pm 53 \\
3,294 \pm 49 \\
3,103 \pm 56 \\
3,266 \pm 57 \\
3,090 \pm 56 \\
2,953 \pm 52 \\
2,931 \pm 51\end{array}$ & $\begin{array}{l}151 \\
181 \\
107 \\
279 \\
165 \\
290 \\
202 \\
250 \\
146 \\
162 \\
209 \\
205 \\
267 \\
274\end{array}$ \\
\hline $\begin{array}{l}\text { GJ } 871.1 \mathrm{~B} \\
N=15 \\
\mathrm{RV}=2,031 \pm 44 \\
\sigma=168.83\end{array}$ & $\begin{array}{l}53327.211 \\
53328.188 \\
53329.195 \\
53522.587\end{array}$ & $\begin{array}{l}2,151 \pm 63 \\
2,031 \pm 65 \\
2,311 \pm 76 \\
1,872 \pm 62\end{array}$ & $\begin{array}{l}244 \\
229 \\
170 \\
228\end{array}$ \\
\hline
\end{tabular}


Table 3

(Continued)

\begin{tabular}{|c|c|c|c|}
\hline \multirow[t]{12}{*}{ Star } & HJD - 2,400,000 & $\begin{array}{l}\text { Radial Velocity } \\
\qquad\left(\mathrm{m} \mathrm{s}^{-1}\right)\end{array}$ & $\mathrm{S} / \mathrm{N}$ \\
\hline & 53523.609 & $1,834 \pm 71$ & 187 \\
\hline & 53596.436 & $2,125 \pm 65$ & 203 \\
\hline & 53597.397 & $2,017 \pm 68$ & 189 \\
\hline & 53669.272 & $1,722 \pm 79$ & 151 \\
\hline & 53686.236 & $2,298 \pm 50$ & 181 \\
\hline & 53928.525 & $2,069 \pm 61$ & 242 \\
\hline & 53930.532 & $2,164 \pm 66$ & 204 \\
\hline & 54308.428 & $2,056 \pm 77$ & 160 \\
\hline & 54309.422 & $2,053 \pm 58$ & 277 \\
\hline & 54311.419 & $1,923 \pm 59$ & 264 \\
\hline & 54312.418 & $1,846 \pm 58$ & 277 \\
\hline \multirow{14}{*}{$\begin{array}{l}\mathrm{HIP} 12545 \\
N=14 \\
\overline{\mathrm{RV}}=8,253 \pm 48 \\
\sigma=179.25\end{array}$} & 53328.384 & $8,407 \pm 54$ & 219 \\
\hline & 53329.401 & $8,399 \pm 73$ & 100 \\
\hline & 53421.208 & $8,422 \pm 60$ & 152 \\
\hline & 53422.205 & $8,201 \pm 54$ & 202 \\
\hline & 53669.402 & $7,945 \pm 55$ & 193 \\
\hline & 53670.393 & $8,145 \pm 54$ & 195 \\
\hline & 53686.263 & $8,219 \pm 55$ & 166 \\
\hline & 53686.519 & $8,077 \pm 54$ & 182 \\
\hline & 53742.255 & $8,438 \pm 62$ & 160 \\
\hline & 53744.203 & $8,495 \pm 48$ & 140 \\
\hline & 53928.614 & $7,985 \pm 65$ & 129 \\
\hline & 53929.608 & $8,116 \pm 50$ & 295 \\
\hline & 53930.592 & $8,325 \pm 53$ & 211 \\
\hline & 53931.641 & $8,372 \pm 55$ & 213 \\
\hline \multirow{13}{*}{$\begin{array}{l}\text { TWA } 7 \\
N=13 \\
\mathrm{RV}=12,446 \pm 26 \\
\sigma=94.18\end{array}$} & 53327.632 & $12,419 \pm 51$ & 197 \\
\hline & 53328.630 & $12,406 \pm 47$ & 183 \\
\hline & 53329.631 & $12,368 \pm 57$ & 121 \\
\hline & 53419.453 & $12,349 \pm 52$ & 195 \\
\hline & 53420.469 & $12,445 \pm 48$ & 291 \\
\hline & 53421.436 & $12,495 \pm 50$ & 232 \\
\hline & 53422.442 & $12,513 \pm 51$ & 204 \\
\hline & 53480.294 & $12,720 \pm 50$ & 220 \\
\hline & 53686.641 & $12,398 \pm 52$ & 158 \\
\hline & 53741.575 & $12,402 \pm 52$ & 180 \\
\hline & 53742.546 & $12,454 \pm 52$ & 187 \\
\hline & 53743.549 & $12,421 \pm 51$ & 188 \\
\hline & 53744.523 & $12,403 \pm 48$ & 220 \\
\hline \multirow{15}{*}{$\begin{array}{l}\text { TWA } 8 \mathrm{~A} \\
N=15 \\
\overline{\mathrm{RV}}=8,679 \pm 23 \\
\sigma=90.07\end{array}$} & 53327.636 & $8,610 \pm 52$ & 175 \\
\hline & 53328.653 & $8,688 \pm 51$ & 192 \\
\hline & 53329.635 & $8,705 \pm 57$ & 124 \\
\hline & 53419.473 & $8,555 \pm 50$ & 243 \\
\hline & 53420.484 & $8,542 \pm 52$ & 183 \\
\hline & 53421.460 & $8,667 \pm 50$ & 225 \\
\hline & 53422.462 & $8,647 \pm 48$ & 208 \\
\hline & 53480.306 & $8,877 \pm 51$ & 217 \\
\hline & 53522.258 & $8,599 \pm 49$ & 186 \\
\hline & 53523.248 & $8,642 \pm 51$ & 214 \\
\hline & 53686.645 & $8,701 \pm 50$ & 195 \\
\hline & 53741.605 & $8,822 \pm 50$ & 231 \\
\hline & 53742.590 & $8,729 \pm 50$ & 252 \\
\hline & 53743.562 & $8,672 \pm 49$ & 242 \\
\hline & 53744.548 & $8,731 \pm 49$ & 257 \\
\hline \multirow{11}{*}{$\begin{array}{l}\text { TWA } 8 \mathrm{~B} \\
N=15 \\
\overline{\mathrm{RV}}=8,607 \pm 32 \\
\sigma=123.46\end{array}$} & 53327.640 & $8,489 \pm 62$ & 130 \\
\hline & 53328.650 & $8,523 \pm 63$ & 126 \\
\hline & 53329.639 & $8,453 \pm 63$ & 122 \\
\hline & 53419.480 & $8,572 \pm 57$ & 170 \\
\hline & 53420.487 & $8,537 \pm 57$ & 162 \\
\hline & 53421.462 & $8,575 \pm 60$ & 140 \\
\hline & 53422.465 & $8,523 \pm 51$ & 170 \\
\hline & 53480.310 & $8,813 \pm 54$ & 197 \\
\hline & 53522.266 & $8,537 \pm 58$ & 164 \\
\hline & 53523.252 & $8,477 \pm 53$ & 219 \\
\hline & 53686.649 & $8,633 \pm 55$ & 164 \\
\hline
\end{tabular}

Table 3

(Continued)

\begin{tabular}{llll}
\hline \hline Star & HJD $-2,400,000$ & Radial Velocity & S/N
\end{tabular}
$\left(\mathrm{m} \mathrm{s}^{-1}\right)$

\begin{tabular}{lll}
\hline 53741.610 & $8,718 \pm 54$ & 202 \\
53742.594 & $8,761 \pm 55$ & 194 \\
53743.567 & $8,662 \pm 54$ & 197 \\
53744.555 & $8,833 \pm 51$ & 253 \\
\hline
\end{tabular}

\begin{tabular}{llll}
\hline TWA 9A & 53419.487 & $11,569 \pm 55$ & 181 \\
$N=11$ & 53420.493 & $11,633 \pm 54$ & 191
\end{tabular}

$\mathrm{RV}=11,649 \pm 21 \quad 53421.466 \quad 11,574 \pm 53 \quad 155$

$\begin{array}{llll}\sigma=70.61 & 53422.469 & 11,567 \pm 57 & 159\end{array}$

$53480.315 \quad 11,716 \pm 54 \quad 184$

$53523.227 \quad 11,659 \pm 55 \quad 173$

$53741.616 \quad 11,750 \pm 55 \quad 175$

$53742.600 \quad 11,569 \pm 57 \quad 164$

$53743.572 \quad 11,686 \pm 57 \quad 179$

\begin{tabular}{llll} 
& 53744.577 & $11,734 \pm 52$ & 215 \\
& 54962.239 & $11,680 \pm 54$ & 198 \\
\hline TWA 9B & 53419.492 & $12,271 \pm 58$ & 150
\end{tabular}

$\begin{array}{llll}N=11 & 53420.497 & 12,320 \pm 54 & 175\end{array}$

$\mathrm{RV}=12,279 \pm 27 \quad 53421.470 \quad 12,101 \pm 58 \quad 144$

$\begin{array}{llll}\sigma=90.25 & 53422.472 & 12,199 \pm 56 & 160\end{array}$

\begin{tabular}{|c|c|}
\hline & 3042.41 \\
\hline
\end{tabular}

$53523.232 \quad 12,367 \pm 56 \quad 168$

$\begin{array}{lll}53741.621 & 12,335 \pm 57 & 156\end{array}$

$53742.605 \quad 12,282 \pm 53 \quad 200$

$53743.577 \quad 12,186 \pm 52 \quad 201$

$\begin{array}{lll}53744.584 & 12,247 \pm 51 & 229\end{array}$

$54962.245 \quad 12,380 \pm 51 \quad 226$

\begin{tabular}{lrrr} 
& 54962.245 & $12,380 \pm 51$ & 226 \\
\hline TWA 11B & 53420.507 & $9,073 \pm 58$ & 156
\end{tabular}

\begin{tabular}{lll}
$N=11$ & 53420.507 & $9,073 \pm 58$ \\
\hline
\end{tabular}

$\begin{array}{llll}\mathrm{RV}=8,923 \pm 58 & 53421.487 & 8,782 \pm 55 & 191 \\ \sigma & 5,719 \pm 55 & 184\end{array}$

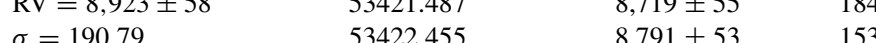

$\begin{array}{llll}\sigma=190.79 & 53422.455 & 8,791 \pm 53 & 153\end{array}$

$53480.334 \quad 8,564 \pm 54 \quad 194$

$53523.243 \quad 9,177 \pm 57 \quad 171$

$53741.633 \quad 8,992 \pm 55 \quad 183$

$\begin{array}{lll}53741.633 & 8,992 \pm 55 & 183 \\ 53742.618 & 9,092 \pm 56 & 186\end{array}$

$53743.592 \quad 8,861 \pm 55 \quad 196$

$\begin{array}{lll}53744.634 & 9,056 \pm 47 & 198\end{array}$

\begin{tabular}{lrrr} 
& 54963.240 & $9,043 \pm 53$ & 219 \\
\hline TWA 12 & 53328.641 & $12,276 \pm 54$ & 255 \\
$N=12$ & 53329.646 & $12,456 \pm 64$ & 163
\end{tabular}

$N=12 \quad 53329.646 \quad 12,456 \pm 64 \quad 163$

$\begin{array}{llll}\mathrm{RV}=12,498 \pm 52 & 53419.457 & 12,352 \pm 61 & 188\end{array}$

$\begin{array}{llll}\sigma=180.79 & 53420.479 & 12,708 \pm 57 & 209\end{array}$

$\begin{array}{lll}53420.479 & 12,708 \pm 57 & 209 \\ 53421.450 & 12,420 \pm 56 & 228\end{array}$

$\begin{array}{lll}53421.450 & 12,420 \pm 56 & 228 \\ 53422.459 & 12,445 \pm 60 & 188\end{array}$

$\begin{array}{lll}53480.297 & 12,302 \pm 60 & 186\end{array}$

$\begin{array}{lll}53480.297 & 12,445 \pm 58 & 196\end{array}$

$\begin{array}{lll}53741.592 & 12,370 \pm 60 & 188\end{array}$

$\begin{array}{lll}53742.557 & 12,655 \pm 60 & 196\end{array}$

$\begin{array}{lll}53742.557 & 12,655 \pm 60 & 196 \\ 53743.558 & 12,758 \pm 59 & 192\end{array}$

\begin{tabular}{lll}
53743.558 & $12,758 \pm 59$ & 192 \\
53744.530 & $12,789 \pm 62$ & 190 \\
\hline
\end{tabular}

\begin{tabular}{llll}
\hline TWA 13A & 53327.646 & $11,889 \pm 57$ & 158 \\
$N=14$ & 53328.670 & $12,220 \pm 55$ & 181 \\
$\mathrm{RV}=11,668 \pm 65$ & 53419.463 & $11,817 \pm 53$ & 218 \\
$\sigma=241.66$ & 53420.472 & $11,488 \pm 56$ & 172 \\
& 53421.454 & $11,469 \pm 55$ & 181 \\
& 53422.445 & $11,523 \pm 56$ & 175 \\
& 53480.303 & $11,559 \pm 54$ & 190 \\
& 53686.656 & $11,616 \pm 55$ & 171 \\
& 53741.598 & $11,365 \pm 58$ & 163 \\
& 53742.549 & $11,324 \pm 56$ & 187 \\
& 53743.551 & $11,901 \pm 55$ & 200 \\
& 53744.537 & $11,735 \pm 53$ & 226 \\
& 54962.223 & $11,752 \pm 49$ & 176 \\
& 54963.225 & $11,696 \pm 50$ & 211
\end{tabular}


Table 3

(Continued)

\begin{tabular}{|c|c|c|c|}
\hline Star & $\mathrm{HJD}-2,400,000$ & $\begin{array}{l}\text { Radial Velocity } \\
\qquad\left(\mathrm{m} \mathrm{s}^{-1}\right)\end{array}$ & $\mathrm{S} / \mathrm{N}$ \\
\hline $\begin{array}{l}\text { TWA 13B } \\
N=13 \\
\overline{\mathrm{RV}}=12,075 \pm 41 \\
\sigma=149.06\end{array}$ & $\begin{array}{l}53327.650 \\
53328.673 \\
53419.468 \\
53420.475 \\
53421.457 \\
53422.448 \\
53480.301 \\
53686.659 \\
53741.601 \\
53742.552 \\
53743.554 \\
53744.542 \\
54962.235\end{array}$ & $\begin{array}{l}12,035 \pm 56 \\
11,677 \pm 58 \\
12,130 \pm 53 \\
11,984 \pm 53 \\
12,071 \pm 54 \\
12,085 \pm 55 \\
12,302 \pm 54 \\
12,020 \pm 54 \\
12,260 \pm 53 \\
12,111 \pm 56 \\
12,050 \pm 54 \\
12,134 \pm 53 \\
12,109 \pm 53\end{array}$ & $\begin{array}{l}167 \\
150 \\
209 \\
214 \\
186 \\
182 \\
190 \\
179 \\
206 \\
206 \\
194 \\
217 \\
217\end{array}$ \\
\hline $\begin{array}{l}\text { TWA } 23 \\
N=14 \\
\overline{\mathrm{RV}}=6,520 \pm 648 \\
\sigma=2425.03\end{array}$ & $\begin{array}{l}53419.500 \\
53420.503 \\
53421.439 \\
53421.483 \\
53422.451 \\
53480.324 \\
53523.238 \\
53686.669 \\
53741.628 \\
53742.613 \\
53743.585 \\
53744.596 \\
54962.254 \\
54963.233\end{array}$ & $\begin{array}{l}4,445 \pm 61 \\
4,504 \pm 62 \\
4,741 \pm 65 \\
4,573 \pm 63 \\
4,778 \pm 53 \\
7,155 \pm 58 \\
8,271 \pm 56 \\
9,022 \pm 62 \\
9,151 \pm 62 \\
9,242 \pm 62 \\
9,204 \pm 60 \\
9,298 \pm 52 \\
3,085 \pm 60 \\
3,816 \pm 63\end{array}$ & $\begin{array}{l}244 \\
230 \\
204 \\
221 \\
243 \\
225 \\
244 \\
196 \\
207 \\
206 \\
224 \\
262 \\
253 \\
231\end{array}$ \\
\hline
\end{tabular}

Note. Reported values are Heliocentric radial velocities.

are listed in the third column. The final column lists the $\mathrm{S} / \mathrm{N}$ (Table 3).

\section{REFERENCES}

Abranin, E. P., Alekseev, I. Y., Avgoloupis, S., et al. 1998, Astron. Astrophys. Trans., 17, 221

Adams, F. C., \& Laughlin, G. 2003, Icarus, 163, 290

Alibert, Y., Mordasini, C., Benz, W., \& Winisdoerffer, C. 2005, A\&A, 434, 343 Baliunas, S. L., Donahue, R. A., Soon, W. H., et al. 1995, ApJ, 438, 269

Baraffe, I., Charbrier, G., Allard, F., \& Hauschildt, P. H. 1998, A\&A, 337, 403 Bean, J. L., Seifahrt, A., Hartmann, H., et al. 2010, ApJ, 713, 410

Blake, C., Charbonneau, D., \& White, R. J. 2010, ApJ, 723, 684

Blake, C., Charbonneau, D., White, R. J., Marley, M. S., \& Saumon, D. 2007, ApJ, 666, 1198

Boss, A. P. 1997, Science, 276, 1836

Boss, A. P. 2004, ApJ, 610, 456

Boss, A. P. 2006, ApJ, 644, 79

Briceño, C., Vivas, A. K., Calvet, N., et al. 2001, Science, 291, 93

Butler, R. P., Johnson, J. A., Marcy, G. W., et al. 2006, PASP, 118, 1685

Butler, R. P., Marcy, G. W., Williams, E., et al. 1996, PASP, 108, 500

Carpenter, J. M., Mamajek, E. E., Hillenbrand, L. A., \& Meyer, M. R. 2006, ApJ, 651,49

Cochran, W. D. 1988, ApJ, 334, 349

Cochran, W. D., Hatzes, A. P., Butler, R. P., \& Marcy, G. W. 1997, ApJ, 483, 457

Crockett, C. J., Mahmud, N. I., Prato, L., et al. 2011, ApJ, 735, 78

Cumming, A., Butler, R. P., Marcy, G. W., et al. 2008, PASP, 120, 531

Delfosse, X., Forveille, T., Perrier, C., \& Mayor, M. 1998, A\&A, 331, 581

Deming, D., Brown, T. M., Charbonneau, D., Harrington, J., \& Richardson, L. J. 2005, ApJ, 622, 1149

Endl, M., Cochran, W. D., Tull, R. G., \& MacQueen, P. J. 2006, AJ, 132, 2755

Feigelson, E. D., Lawson, W. A., Stark, M., Townsley, L., \& Garmire, G. P. 2006, AJ, 131, 1730

Figueira, P., Marmier, M., Bonfils, X., et al. 2010, A\&A, 513, 8

Goldreich, P., \& Tremaine, S. 1980, ApJ, 241, 425
Haisch, K., Lada, E. A., \& Lada, C. J. 2001, ApJ, 553, 153

Hauschildt, P. H., Allard, F., \& Baron, E. 1999, ApJ, 512, 377

Hawley, S. L., Gizis, J. E., \& Reid, I. N. 1996, AJ, 112, 2799

Hebb, L., Petro, L., Ford, H. C., et al. 2007, MNRAS, 379, 63

Henry, T. J., Kirkpatrick, J. D., \& Simons, D. A. 1994, AJ, 108, 1437

Henry, T. J., \& McCarthy, D. W., Jr. 1993, AJ, 106, 773

Herbst, W., Herbst, D. K., Grossman, E. J., \& Weinstein, D. 1994, AJ, 108, 1906

Hernán-Obispo, M., Gálvez-Ortiz, M. C., Anglada-Escudé, G., et al. 2010, A\&A, 512, 45

Hillenbrand, L. A., \& White, R. J. 2004, ApJ, 604, 741

Horne, K. 1986, PASP, 98, 609

Howard, A. W., Marcy, G. W., Johnson, J. A., et al. 2010, Science, 330, 653

Huélamo, N., Figueira, P., Bonfils, X., et al. 2008, A\&A, 489, 9

Huerta, M., Johns-Krull, C. M., Prato, L., Hartigan, P., \& Jaffe, D. T. 2008, ApJ, 678,472

Hünsch, M., Schmitt, J. H. M. M., Sterzik, M. F., \& Voges, W. 1999, A\&AS, 135,319

Ireland, M. J., Kraus, A., Martinache, F., Law, N., \& Hillenbrand, L. A. 2011, ApJ, 726, 113

Johns-Krull, C. M., \& Valenti, J. A. 1996, ApJ, 459, L95

Kalas, P., Graham, J. R., Chiang, E., et al. 2008, Science, 322, 1345

Kalas, P., Liu, M. C., \& Matthews, B. C. 2004, Science, 303, 1990

Kasper, M., Apai, D., Janson, M., \& Brandner, W. 2007, A\&A, 472, 321

Kataria, T., \& Simon, M. 2010, AJ, 140, 206

Kennedy, G. M., \& Kenyon, S. J. 2008, ApJ, 673, 502

Kraus, A. L., \& Hillenbrand, L. A. 2007, AJ, 134, 2340

Lagrange, A.-M., Bonnefoy, M., Chauvin, G., et al. 2010, Science, 329, 57

Latham, D. W., Stefanik, R. P., Mazeh, T., Mayor, M., \& Burki, G. 1989, Nature, 339,38

Lawson, W. A., \& Crause, L. A. 2005, MNRAS, 357, 1399

Leggett, S. 1992, ApJS, 82, 351

Lin, D. N. C., Bodenheimer, P., \& Richardson, D. C. 1996, Nature, 380, 606

Livingston, W., \& Wallace, L. 1991, Atlas of the Solar Spectrum in the Infrared from 1850 to $9000 \mathrm{~cm}^{-1}$ (NSO Technical Report; Tucson, AZ: NSO)

Lubow, S. H., \& Ida, S. 2010, in Exoplanets, ed. S. Seager (Tucson, AZ: Univ. Arizona Press), 347

Luhman, K. 2003, ApJ, 593, 1093

Macintosh, B. A., Graham, J. R., Palmer, D. W., et al. 2008, Proc. SPIE, 7015, 180

Mahmud, N., Crockett, C. J., Johns-Krull, C. M., et al. 2011, ApJ, 736, 123

Malmberg, D., \& Davies, M. B. 2009, MNRAS, 394, 26

Malmberg, D., de Angeli, F., Davies, M. B., et al. 2007, MNRAS, 378, 1207

Marcy, G. W., \& Butler, R. P. 1996, ApJ, 464, 147

Marois, C., Macintosh, B., Barman, T., et al. 2008, Science, 322, 1348

Mathieu, R. D., Baraffe, I., Simon, M., Stassun, K. G., \& White, R. J. 2007, in Protostars and Planets V, ed. B. Reipurth, D. Jewitt, \& K. Keil (Tucson, AZ: Univ. Arizona Press), 411

Mathioudakis, M., Fruscione, A., Drake, J. J., et al. 1995, A\&A, 300, 775

Mayor, M., \& Queloz, D. 1995, Nature, 378, 355

Mayer, L., Quinn, T., Wadsley, J., \& Stadel, J. 2002, Science, 298, 1756

McLean, I. S., Becklin, E. E., Bendiksen, O., et al. 1998, Proc. SPIE, 3354 566

Mentuch, E., Brandeker, A., van Kerkwijk, M. H., Jayawardhana, R., \& Hauschildt, P. H. 2008, ApJ, 689, 1127

Meru, F., \& Bate, M. R. 2010, MNRAS, 406, 2279

Meru, F., \& Bate, M. R. 2011, MNRAS, 411, 1

Mizuno, H. 1980, Prog. Theor. Phys., 64, 544

Muirhead, P. S., Edelstein, J., Erskine, D. J., et al. 2011, PASP, in press

Nidever, D. L., Marcy, G. W., Butler, R. P., Fischer, D. A., \& Vogt, S. S. 2002, ApJS, 141, 503

Nelder, J. A., \& Mead, R. 1965, Comput. J., 7, 308

Neuhäuswer, R., Guenther, E. W., Wuchterl, G., et al. 2005, A\&A, 435, 13

Norton, A. J., Wheatley, P. J., West, R. G., et al. 2007, A\&A, 476, 785

Osten, R. A., Hawley, S. L., Allred, J., et al. 2006, ApJ, 647, 1349

Pascucci, I., Gorti, U., Hollenbach, D., et al. 2006, ApJ, 651, 1177

Paulson, D. B., \& Yelda, S. 2006, PASP, 118, 706

Pettersen, B. R., Evans, D. S., \& Coleman, L. A. 1984, ApJ, 282, 214

Piskunov, N. E., \& Valenti, J. A. 2002, A\&A, 385, 1095

Pizzolato, N., Maggio, A., Micela, G., Sciortino, S., \& Ventura, P. 2003, A\&A, 397,147

Pollack, J. B., Hubickyj, O., Bodenheimer, P., et al. 1996, Icarus, 124, 62

Pont, F., Hébrard, G., Irwin, J. M., et al. 2009, A\&A, 502, 695

Prato, L., Huerta, M., Johns-Krull, C. M., et al. 2008, ApJ, 687, 103

Press, W. H., Teulolsky, S. A., Vetterling, W. T., \& Flannery, B. P. 1992, Numerical Recipes in C. The Art of Scientific Computing (2nd ed.; Cambridge: Cambridge Univ. Press)

Queloz, D., Henry, G. W., Sivan, J. P., et al. 2001, A\&A, 379, 279 
Raymond, S. N., Armitage, P. J., Moro-Martín, A., et al. 2011, A\&A, 530, 62 Reiners, A. 2009, A\&A, 498, 853

Reiners, A., \& Basri, G. 2007, AJ, 656, 1121

Reiners, A., Bean, J. L., Huber, K. F., et al. 2010, ApJ, 710, 432

Robinson, S. E., Laughlin, G., Bodenheimer, P., \& Fischer, D. 2006, ApJ, 643, 484

Saar, S. H., \& Donahue, R. A. 1997, ApJ, 485, 319

Sato, B., Fischer, D. A., Henry, G. W., et al. 2005, ApJ, 633, 465

Scholz, A., Coffey, J., Brandeker, A., \& Jayawardhana, R. 2007, ApJ, 662, 1254

Seifahrt, A., \& Käufl, H. U. 2008, A\&A, 491, 929

Seifahrt, A., Käufl, H. U., Zängl, G., et al. 2010, A\&A, 524, 11

Setiawan, J., Henning, Th., Launhardt, R., et al. 2008, Nature, 451, 38

Siess, L., Dufour, E., \& Forestini, M. 2000, A\&A, 358, 593
Song, I., Bessell, M. S., \& Zuckerman, B. 2003, ApJ, 581, 43

Song, I., Zuckerman, B., \& Bessell, M. S. 2002, ApJ, 599, 342

Stamatellos, D., \& Whitworth, A. P. 2008, A\&A, 480, 879

Stauffer, J. R., Caillault, J.-P., Gagné, M., Prosser, C. F., \& Hartmann, L. W. 1994, ApJS, 91, 625

Torres, C. A. O., Quast, G. R., Melo, C. H. F., \& Sterzik, M. F. 2008, in Handbook of Star Forming Regions, Volume II: The Southern Sky', ed. B. Reipurth (San Francisco, CA: ASP Monograph Publications, Vol. 5), 757 Udry, S., \& Santos, N. C. 2007, ARA\&A, 45, 397

Walker, G. A. H., Walker, A. R., Irwin, A. W., et al. 1995, Icarus, 116, 359

Webb, R. A., Zuckerman, B., Platais, I., et al. 1999, ApJ, 512, 63

Wright, J. T., Fakhouri, O., Marcy, G. W., et al. 2010, PASP, 123, 412

Zuckerman, B., \& Song, I. 2004, ARA\&A, 42, 685 